\title{
LIMIT CYCLES COMING FROM SOME UNIFORM ISOCHRONOUS CENTERS
}

\author{
HAIHUA LIANG, JAUME LLIBRE, AND JOAN TORREGROSA
}

\begin{abstract}
This article is about the weak 16-th Hilbert problem, i.e. we analyze how many limit cycles can bifurcate from the periodic orbits of a given polynomial differential center when it is perturbed inside a class of polynomial differential systems. More precisely, we consider the uniform isochronous centers

$$
\dot{x}=-y+x^{2} y\left(x^{2}+y^{2}\right)^{n}, \quad \dot{y}=x+x y^{2}\left(x^{2}+y^{2}\right)^{n},
$$

of degree $2 n+3$ and we perturb them inside the class of all polynomial differential systems of degree $2 n+3$. For $n=0,1$ we provide the maximum number of limit cycles, 3 and 8 respectively, that can bifurcate from the periodic orbits of these centers using averaging theory of first order, or equivalently Abelian integrals. For $n=2$ we show that at least 12 limit cycles can bifurcate from the periodic orbits of the center.
\end{abstract}

\section{Introduction AND STATEMENT OF THE MAIN RESUltS}

The second part of the 16th Hilbert's problem asks for the maximum number $H(n)$ of limit cycles that planar polynomial differential systems of degree $n$ can have, see for instance $[7,8,11]$, and the references quoted therein. The problem on the number $H(n)$ remains open, even for $n=2$.

A weaker problem than the 16th Hilbert's problem, known now as the weak 16th Hilbert's problem was proposed by Arnold [2], who asked for the maximum number $Z(m, n)$ of isolated zeros of Abelian integrals of all polynomial 1 -form of degree $n$ over algebraic ovals of degree $m$, for more details on the weak 16th Hilbert's problem see [4, 9, 19], and the hundreds of references quoted in these articles. Unfortunately the weak 16th Hilbert's problem is also extremely hard to study. On the other hand, the weak 16th Hilbert's problem is a particular case of the problem of studying the maximum number of limit cycles that can bifurcate from the periodic orbits of a center of a polynomial differential system of degree $m-1$ when it is perturbed inside the class of all polynomial differential systems of degree $n$. Of course $Z(m, n) \leq H(\max (n, m-1))$.

In this paper we provide lower bounds for the maximum number of limit cycles that can bifurcate from the periodic solutions of a polynomial differential uniform isochronous center of degree 3,5 and 7 when it its perturbed inside the class of all polynomial differential systems of the same degree. The main result it is based on the averaging theory of first order. But here the main work is to study the maximum number of simple zeros of the obtained averaged functions, because not always the standard study of Extended Chebyshev systems (ET-systems) can be applied (see Appendix 2). The study is based on some new results that can be applied when the family of functions that define $\mathcal{F}$ is not an ET-system. Some delicate study using qualitative theory on some differential equations is also needed to complete the study.

2010 Mathematics Subject Classification. 37G15, 37C80, 37C30.

Key words and phrases. periodic solution, uniform isochronous centers, averaging theory, weak Hilbert problem. 
More precisely, we consider the polynomial differential system

$$
\begin{aligned}
& \dot{x}=-y+x^{2} y\left(x^{2}+y^{2}\right)^{n}, \\
& \dot{y}=x+x y^{2}\left(x^{2}+y^{2}\right)^{n},
\end{aligned}
$$

of degree $2 n+3$ with $n \geq 0$, having a uniform isochronous center at the origin of coordinates, which in polar coordinates $(r, \theta)$, where $x=r \sin \theta$ and $y=r \cos \theta$, becomes

$$
\begin{aligned}
& \dot{r}=r^{2 n+3} \cos \theta \sin \theta, \\
& \dot{\theta}=1 .
\end{aligned}
$$

Since $\dot{\theta}=1$ the center (1) is a uniform isochronous center, which taking as independent variable the variable $\theta$ writes

$$
\frac{d r}{d \theta}=r^{\prime}=r^{2 n+3} \cos \theta \sin \theta .
$$

An easy computation shows that the periodic solutions $r\left(\theta, r_{0}\right)$ surrounding the center $r=0$ such that $r\left(0, r_{0}\right)=r_{0}$ are

$$
r\left(\theta, r_{0}\right)=r_{0}\left(1-(n+1) r_{0}^{2(n+1)} \sin ^{2} \theta\right)^{-\frac{1}{2 n+2}}
$$

with $0<r_{0}<(n+1)^{-\frac{1}{2 n+2}}$. The global phase portraits, in the Poincaré disc, of system (1) for $n=0,1,2$ are shown in Figure 1.
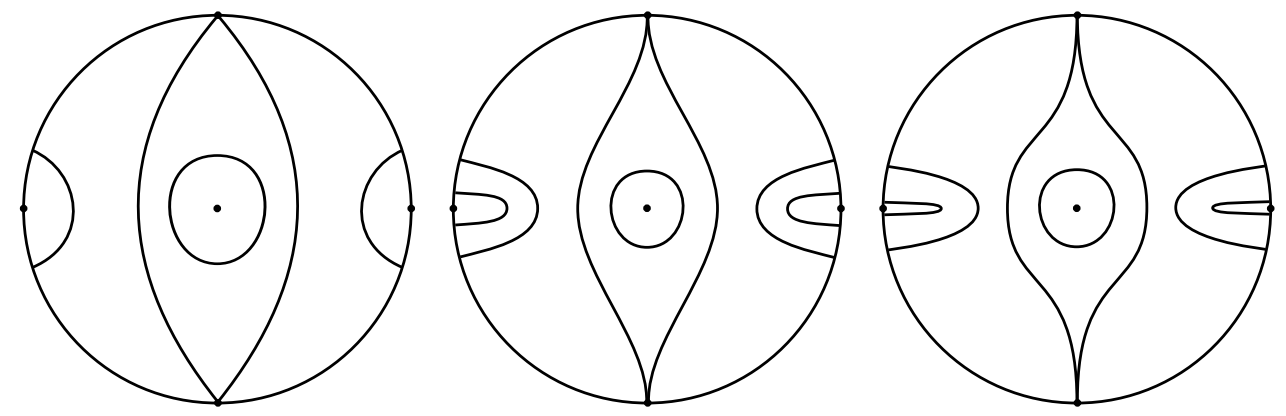

Figure 1. Phase portrait of the uniform isochronous center (1) for $n=0$, $n=1$, and $n=2$, respectively.

Our purpose is to provide a lower bound for the maximum number of limit cycles that can bifurcate from the periodic solutions $r\left(\theta, r_{0}\right)$ surrounding the uniform isochronous center at $r=0$ of degree 3,5,7 when we perturb it inside the class of all polynomial differential systems of degree $3,5,7$, respectively. In other words, we study the number of limit cycles of the following three polynomial differential systems

$$
\begin{gathered}
\dot{x}=-y+x^{2} y+\varepsilon \sum_{i+j=0}^{3} a_{i j} x^{i} y^{j}, \\
\dot{y}=x+x y^{2}+\varepsilon \sum_{i+j=0}^{3} b_{i j} x^{i} y^{j} \\
\dot{x}=-y+x^{2} y\left(x^{2}+y^{2}\right)+\varepsilon \sum_{i+j=0}^{5} a_{i j} x^{i} y^{j}, \\
\dot{y}=x+x y^{2}\left(x^{2}+y^{2}\right)+\varepsilon \sum_{i+j=0}^{5} b_{i j} x^{i} y^{j}
\end{gathered}
$$


and

$$
\begin{aligned}
& \dot{x}=-y+x^{2} y\left(x^{2}+y^{2}\right)^{2}+\varepsilon \sum_{i+j=0}^{7} a_{i j} x^{i} y^{j}, \\
& \dot{y}=x+x y^{2}\left(x^{2}+y^{2}\right)^{2}+\varepsilon \sum_{i+j=0}^{7} b_{i j} x^{i} y^{j},
\end{aligned}
$$

where $\varepsilon$ is a small parameter.

Our main result is the following.

Theorem 1.1. For $|\varepsilon| \neq 0$ sufficiently small using averaging theory of first order we obtain that

(a) system (3) can have 0,1,2,3 limit cycles and no more;

(b) system (4) can have $0,1,2, \ldots, 8$ limit cycles and no more;

(c) system (5) can have $0,1,2, \ldots, 12$ limit cycles.

In fact in the plane $\mathbb{R}^{2}$ the averaging theory of first order, or the generalized Abelian integrals, or the Melnikov function provide the same information because all these methods are based on the study of the first term in $\varepsilon$ of the Poincaré return map. Some concrete applications of that theory to planar differential systems of low degree can be seen in $[6,16]$. In higher dimension, the averaging theory can be also used, for example, for the study of the Hopf bifurcation, see $[12,13]$.

As we will see, by using the averaging theory of first order, the limit cycles of the perturbed system, which emerge from the period annulus of the isochronous center of system (1), correspond to the zeros of a linear combination of the functions $f_{0}, f_{1}, \ldots, f_{\left(n^{2}+7 n+6\right) / 2}$, $n=0,1,2$. The proof of Theorem 1.1 for the case $n=0$ is easy and it is done in Section 2. But the difficulty arises evidently as $n$ increases. For $n=1$, as the collection of functions $f_{0}, \ldots, f_{7}$ is not an ET-system, part of our efforts has been focused on determining the numbers of simple zeros of Wronskian determinants $W_{6}(s)$ and $W_{7}(s)$, which have the expressions $\sum_{i=0}^{k} a_{i}(s) E^{i}(s) K^{k-i}(s)(k=2,3)$, where $a_{i}$ is a polynomial of high degree, $E$ and $K$ are respectively the elliptic integrals of the first kind and second kind:

$$
E(x)=\int_{0}^{\pi / 2} \sqrt{1-x \sin ^{2} \theta} d \theta, K(x)=\int_{0}^{\pi / 2} \frac{1}{\sqrt{1-x \sin ^{2} \theta}} d \theta .
$$

The proof is done using qualitative analysis and algebraic calculations. It turns out that all the Wronskian determinants but $W_{6}(s)$ do not vanish and the later has a unique zero which is simple. So the conditions of the classic Chebyshev criterion are not satisfied. According to the result of the recent paper [15], the maximum number of zeros of the linear combination of $f_{0}, \ldots, f_{7}$ is less than or equal to 8 . Consequently, another part of our efforts have been focused on proving that the possible upper bound 8 can be reached. To show this, we construct a function which has a zero of multiplicity 7 as well as an extra simple zero. Then, under suitable perturbation this function possesses 8 simple zeros. This is done in Section 3. For $n=2$, the corresponding functions $f_{0}, f_{1}, \ldots, f_{12}$, which contain several hypergeometric functions, is neither an Extended Complete Chebyshev system, nor a system satisfying the condition of [15]. We do not know how to find out the maximum number of zeros of all the possible linear combination of $f_{0}, f_{1}, \ldots, f_{12}$. Instead, we provide a lower bound for this number or zeros. This is done in Section 4 .

\section{Proof of Statement $(a)$ of Theorem 1.1}

This section is devoted to the proof of statement $(a)$ of Theorem 1.1 by using Theorem 4.3 (see Appendix 1). 
First, we make the polar coordinate transformation and change system (3) to

$$
\frac{d r}{d \theta}=R_{0}(\theta, r)+\varepsilon R_{1}(\theta, r)+O\left(\varepsilon^{2}\right)
$$

where $R_{0}(\theta, r)=r^{3} \cos \theta \sin \theta$ and

$$
\begin{aligned}
R_{1}(\theta)= & a_{00} C+b_{00} S+r\left[a_{10} C^{2}+\left(a_{01}+b_{10}\right) C S+b_{01} S^{2}\right]+r^{2}\left[a_{20} C^{3}\right. \\
& \left.+\left(a_{11}-b_{00}+b_{20}\right) C^{2} S+\left(a_{00}+a_{02}+b_{11}\right) C S^{2}+b_{02} S^{3}\right] \\
& +r^{3}\left[a_{30} C^{4}+\left(a_{21}-b_{10}+b_{30}\right) C^{3} S+\left(a_{10}+a_{12}-b_{01}+b_{21}\right) C^{2} S^{2}\right. \\
& \left.+\left(a_{01}+a_{03}+b_{12}\right) C S^{3}+b_{03} S^{4}\right]+r^{4}\left[-b_{20} C^{4} S+\left(a_{20}-b_{11}\right) C^{3} S^{2}\right. \\
& \left.+\left(a_{11}-b_{02}\right) C^{2} S^{3}+a_{02} C S^{4}\right]+r^{5}\left[-b_{30} C^{5} S+\left(a_{30}-b_{21}\right) C^{4} S^{2}\right. \\
& \left.+\left(a_{21}-b_{12}\right) C^{3} S^{3}+\left(a_{12}-b_{03}\right) C^{2} S^{4} a_{03} C S^{5}\right]
\end{aligned}
$$

with $C=\cos \theta$ and $S=\sin \theta$.

Since equation $(6)_{\varepsilon=0}$ has the periodic solutions $r\left(\theta, r_{0}\right)$ satisfying $r_{0}=r\left(0, r_{0}\right)$ for $0<$ $r_{0}<1$ given in (2), according to the averaging theory described in Appendix 1, we solve the variational differential equation

$$
\frac{d M}{d \theta}=\frac{\partial}{\partial r} R_{0}\left(\theta, r\left(\theta, r_{0}\right)\right) M
$$

with $M_{r_{0}}(0)=1$ and get the fundamental solution

$$
M_{r_{0}}(\theta)=\left(1-r_{0}^{2} \sin ^{2} \theta\right)^{-3 / 2} .
$$

Next we go to study the maximum number of zeros of the function

$$
\mathcal{F}\left(r_{0}\right)=\int_{0}^{2 \pi} M_{r_{0}}^{-1}(\theta) R_{1}\left(\theta, r\left(\theta, r_{0}\right)\right) d \theta, \quad \text { with } \quad r_{0} \in(0,1) .
$$

Using expression (7), we perform the computation and we obtain

$$
\begin{aligned}
\mathcal{F}\left(r_{0}\right)= & \frac{\pi}{r_{0}}\left(\left(a_{10}-a_{12}+3 a_{30}+b_{01}+b_{03}-3 b_{21}\right) r_{0}^{2}+\left(b_{21}+b_{03}-b_{01}\right) r_{0}^{4}\right. \\
& \left.+2\left(a_{12}-a_{30}-b_{03}+b_{21}\right)\left(1-\sqrt{1-r_{0}^{2}}\right)-2\left(a_{30}-b_{21}\right) r_{0}^{2} \sqrt{1-r_{0}^{2}}\right) .
\end{aligned}
$$

Denoting

$$
\begin{aligned}
& \alpha_{0}=\pi\left(a_{10}-a_{12}+3 a_{30}+b_{01}+b_{03}-3 b_{21}\right), \\
& \alpha_{1}=\pi\left(b_{21}+b_{03}-b_{01}\right), \\
& \alpha_{2}=2 \pi\left(a_{12}-a_{30}-b_{03}+b_{21}\right), \\
& \alpha_{3}=-2 \pi\left(a_{30}-b_{21}\right),
\end{aligned}
$$

and

$$
f_{0}(s)=1-s^{2}, f_{1}(s)=\left(1-s^{2}\right)^{2}, f_{2}(s)=1-s, f_{3}(s)=s\left(1-s^{2}\right),
$$

where $s=\sqrt{1-r_{0}^{2}} \in(0,1)$. Then

$$
\begin{aligned}
r_{0} \mathcal{F}\left(r_{0}\right) & =\alpha_{0} f_{0}(s)+\alpha_{1} f_{1}(s)+\alpha_{2} f_{2}(s)+\alpha_{3} f_{3}(s) \\
& =(1-s)\left(\alpha_{0}+\alpha_{1}+\alpha_{2}+\left(\alpha_{0}+\alpha_{1}+\alpha_{3}\right) s+\left(\alpha_{3}-\alpha_{1}\right) s^{2}-\alpha_{1} s^{3}\right) .
\end{aligned}
$$

It is not hard to check that $\alpha_{0}, \alpha_{1}, \alpha_{2}$ and $\alpha_{3}$ are independent constants and hence the four numbers $\alpha_{0}+\alpha_{1}+\alpha_{2}, \alpha_{0}+\alpha_{1}+\alpha_{3}, \alpha_{3}-\alpha_{1}$ and $\alpha_{1}$ can be chosen freely. Thus it follows from (8) that $\mathcal{F}\left(r_{0}\right)$ can have $0,1,2,3$ (and no more) simple zeros in the interval $(0,1)$.

Using Theorem 4.3 (see Appendix 1), statement (a) of Theorem 1.1 is proved. 


\section{Proof of statement (b) of Theorem 1.1}

In this section we will study the number of limit cycles of system (4) by using averaging theory of first order. We will only prove that this maximum number is 8 because according to the proof, the reader can easy see that system (4) can have $0,1,2, \ldots, 8$ limit cycles. First, let us state and prove the following lemma.

Lemma 3.1. The maximum number of limit cycles of system (4) which emerge from the period annulus around the center of system $(4)_{\varepsilon=0}$, by using averaging theory of first order, is equal to the maximum number of simple zeros of the function

$$
G(s)=b_{0} f_{0}(s)+b_{1} f_{1}(s)+\cdots+b_{7} f_{7}(s), s \in(0,1),
$$

where $b_{0}, b_{1}, \ldots, b_{7}$ are independent arbitrary constants and

$$
\begin{aligned}
& f_{0}(s)=(1-s)^{2}, \\
& f_{1}(s)=(1-s)\left(1-s^{2}\right), \\
& f_{2}(s)=\left(1-s^{2}\right)^{2}, \\
& f_{3}(s)=(1-s)\left(1-s^{2}\right)^{2}, \\
& f_{4}(s)=\left(1-s^{2}\right)^{3}, \\
& f_{5}(s)=\left(1-s^{2}\right)^{5 / 2} g_{1}(s), \\
& f_{6}(s)=\left(1-s^{2}\right)^{1 / 2}\left(g_{1}(s)-g_{2}(s)\right)-\frac{1}{2}\left(1-s^{2}\right) g_{2}(s), \\
& f_{7}(s)=\left(1-s^{2}\right)^{3 / 2}\left(g_{1}(s)-g_{2}(s)\right),
\end{aligned}
$$

with

$$
g_{1}(s)=2 E\left(1-s^{2}\right), \quad g_{2}(s)=2 s^{2} K\left(1-s^{2}\right) .
$$

Proof. Under the polar coordinate transformation system (4) can be changed to

$$
\frac{d r}{d \theta}=R_{0}(\theta, r)+\varepsilon R_{1}(\theta, r)+O\left(\varepsilon^{2}\right)
$$

where $R_{0}(\theta, r)=r^{5} \cos \theta \sin \theta$ and

$$
\begin{aligned}
R_{1}(\theta)= & \left(a_{00} C+b_{00} S\right)+r\left(a_{10} C^{2}+\left(a_{01}+b_{10}\right) C S+b_{01} S^{2}\right) \\
& \left.+r^{2}\left[a_{20} C^{3}+\left(a_{11}+b_{20}\right) C^{2} S+\left(b_{11}+a_{02}\right) C S^{2}+b_{02}\right) S^{3}\right]+r^{3}\left[a_{30} C^{4}\right. \\
& \left.+\left(a_{21}+b_{30}\right) C^{3} S+\left(a_{12}+b_{21}\right) C^{2} S^{2}+\left(a_{03}+b_{12}\right) C S^{3}+b_{03} S^{4}\right] \\
& +r^{4}\left[a_{40} C^{5}-b_{00} C^{2} S+\left(a_{31}+b_{40}\right) C^{4} S+a_{00} C S^{2}+\left(a_{22}+b_{31}\right) C^{3} S^{2}\right. \\
& \left.+\left(a_{13}+b_{22}\right) C^{2} S^{3}+\left(a_{04}+b_{13}\right) C S^{4}+b_{04} S^{5}\right]+r^{5}\left[a_{50} C^{6}-b_{10} C^{3} S\right. \\
& +\left(a_{41}+b_{50}\right) C^{5} S+\left(a_{10}-b_{01}\right) C^{2} S^{2}+\left(a_{32}+b_{41}\right) C^{4} S^{2}+a_{01} C S^{3} \\
& \left.+\left(a_{23}+b_{32}\right) C^{3} S^{3}+\left(a_{14}+b_{23}\right) C^{2} S^{4}+\left(a_{05}+b_{14}\right) C S^{5}+b_{05} S^{6}\right] \\
& +r^{6}\left[-b_{20} C^{4} S+\left(a_{20}-b_{11}\right) C^{3} S^{2}+\left(a_{11}-b_{02}\right) C^{2} S^{3}+a_{02} C S^{4}\right] \\
& +r^{7}\left[-b_{30} C^{5} S+\left(a_{30}-b_{21}\right) C^{4} S^{2}+\left(a_{21}-b_{12}\right) C^{3} S^{3}+\left(a_{12}-b_{03}\right) C^{2} S^{4}\right. \\
& \left.+a_{03} C S^{5}\right]+r^{8}\left[-b_{40} C^{6} S+\left(a_{40}-b_{31}\right) C^{5} S^{2}+\left(a_{31}-b_{22}\right) C^{4} S^{3}\right. \\
& \left.+\left(a_{22}-b_{13}\right) C^{3} S^{4}+\left(a_{13}-b_{04}\right) C^{2} S^{5}+a_{04} C S^{6}\right] \\
& +r^{9}\left[-b_{50} C^{7} S+\left(a_{50}-b_{41}\right) C^{6} S^{2}+\left(a_{41}-b_{32}\right) C^{5} S^{3}+\left(a_{32}-b_{23}\right) C^{4} S^{4}\right. \\
& \left.+\left(a_{23}-b_{14}\right) C^{3} S^{5}+\left(a_{14}-b_{05}\right) C^{2} S^{6}+a_{05} C S^{7}\right],
\end{aligned}
$$

with $C=\cos \theta, S=\sin \theta$. 
Equation $(12)_{\varepsilon=0}$ has the periodic solutions $r\left(\theta, r_{0}\right)=r_{0}\left(1-2 r_{0}^{4} \sin ^{2} \theta\right)^{-1 / 4}$ satisfying $r_{0}=r\left(0, r_{0}\right)$ for $0<r_{0}<2^{-1 / 4}$. We solve the variational differential equation

$$
\frac{d M}{d \theta}=\frac{\partial}{\partial r} R_{0}\left(\theta, r\left(\theta, r_{0}\right)\right) M
$$

with $M_{r_{0}}(0)=1$ and get the fundamental solution

$$
M_{r_{0}}(\theta)=\left(1-2 r_{0}^{4} \sin ^{2} \theta\right)^{-5 / 4} .
$$

Next, a straightforward calculation leads to

$$
\begin{aligned}
\mathcal{F}\left(r_{0}\right)= & \int_{0}^{2 \pi} M_{r_{0}}^{-1}(\theta) R_{1}\left(\theta, r\left(\theta, r_{0}\right)\right) d \theta \\
= & \int_{0}^{2 \pi} r_{0}\left(1-2 r_{0}^{4} S^{2}\right)\left(c_{00}+c_{02} S^{2}+c_{04} S^{4}+c_{06} S^{6}+c_{40} C^{4}+c_{60} C^{6}\right. \\
& \left.+c_{22} C^{2} S^{2}+c_{42} C^{4} S^{2}+c_{62} C^{6} S^{2}+c_{24} C^{2} S^{4}+c_{44} C^{4} S^{4}+c_{26} C^{2} S^{6}+\Upsilon(C, S)\right) d \theta,
\end{aligned}
$$

for $r_{0} \in\left(0,2^{-1 / 4}\right)$, where $\Upsilon(C, S)=\sum \alpha_{i, j} C^{i} S^{j}$ is a polynomial in $C, S$ with $i$ or $j$ being an odd number, which leads to $\int_{0}^{2 \pi} r_{0}\left(1-2 r_{0}^{4} S^{2}\right) \Upsilon(C, S) d \theta=0$,

$$
\begin{aligned}
& c_{00}=a_{10}:=e_{0}, \\
& c_{02}=-a_{10}+b_{01}:=e_{1}, \\
& c_{04}=b_{03} r^{2}\left(\theta, r_{0}\right):=e_{2} r^{2}\left(\theta, r_{0}\right), \\
& c_{06}=b_{05} r^{4}\left(\theta, r_{0}\right):=e_{3} r^{4}\left(\theta, r_{0}\right), \\
& c_{40}=a_{30} r^{2}\left(\theta, r_{0}\right):=e_{4} r^{2}\left(\theta, r_{0}\right), \\
& c_{60}=a_{50} r^{4}\left(\theta, r_{0}\right):=e_{5} r^{4}\left(\theta, r_{0}\right), \\
& c_{22}=\left(a_{12}+b_{21}\right) r^{2}\left(\theta, r_{0}\right)+\left(a_{10}-b_{01}\right) r^{4}\left(\theta, r_{0}\right):=e_{6} r^{2}\left(\theta, r_{0}\right)-e_{1} r^{4}\left(\theta, r_{0}\right), \\
& c_{24}=\left(a_{14}+b_{23}\right) r^{4}\left(\theta, r_{0}\right)+\left(a_{12}-b_{03}\right) r^{6}\left(\theta, r_{0}\right):=e_{7} r^{4}\left(\theta, r_{0}\right)+e_{8} r^{6}\left(\theta, r_{0}\right), \\
& c_{26}=\left(a_{14}-b_{05}\right) r^{8}\left(\theta, r_{0}\right):=e_{9} r^{8}\left(\theta, r_{0}\right), \\
& c_{42}=\left(a_{32}+b_{41}\right) r^{4}\left(\theta, r_{0}\right)+\left(a_{30}-b_{21}\right) r^{6}\left(\theta, r_{0}\right):=e_{10} r^{4}\left(\theta, r_{0}\right)+e_{11} r^{6}\left(\theta, r_{0}\right), \\
& c_{44}=\left(a_{32}-b_{23}\right) r^{8}\left(\theta, r_{0}\right):=e_{12} r^{8}\left(\theta, r_{0}\right), \\
& c_{62}=\left(a_{50}-b_{41}\right) r^{8}\left(\theta, r_{0}\right):=e_{13} r^{8}\left(\theta, r_{0}\right) .
\end{aligned}
$$

It is not hard to check that the constants $e_{0}, e_{1}, \ldots, e_{13}$ are independent. Computing (13) we get

$$
\mathcal{F}\left(r_{0}\right)=I_{1}\left(r_{0}\right)+I_{2}\left(r_{0}\right)+I_{3}\left(r_{0}\right)+I_{4}\left(r_{0}\right),
$$

where

$$
\begin{aligned}
& I_{1}\left(r_{0}\right)=\alpha_{1} r_{0}+\alpha_{2} r_{0}^{5}, \\
& I_{2}\left(r_{0}\right)=\frac{1}{15 r_{0}^{5}}\left(\left(\alpha_{3}+\alpha_{4} r_{0}^{4}+\alpha_{5} r_{0}^{8}\right) \bar{g}_{1}\left(r_{0}\right)-\left(\alpha_{3}+\left(\alpha_{3}+\alpha_{4}\right) r_{0}^{4}\right) \bar{g}_{2}\left(r_{0}\right)\right), \\
& I_{3}\left(r_{0}\right)=-\frac{\pi}{16 r_{0}^{7}}\left(\left(2 \alpha_{6}+2 \alpha_{7} r_{0}^{4}+\alpha_{8} r_{0}^{8}\right) \bar{g}_{3}\left(r_{0}\right)+\left(2 \alpha_{6} r_{0}^{4}+\left(\alpha_{6}+2 \alpha_{7}\right) r_{0}^{8}\right.\right. \\
& \left.\left.+\left(\alpha_{6}+\alpha_{7}+\alpha_{8}\right) r_{0}^{12}\right)\right) \\
& I_{4}\left(r_{0}\right)=-\frac{1}{30 r_{0}^{5}}\left(\left(4 \alpha_{9}+\alpha_{10} r_{0}^{4}-\left(7 \alpha_{9}+\alpha_{10}\right) r_{0}^{8}\right) \bar{g}_{1}\left(r_{0}\right)-\left(4 \alpha_{9}+\left(4 \alpha_{9}+\alpha_{10}\right) r_{0}^{4}\right) \bar{g}_{2}\left(r_{0}\right)\right) \text {, }
\end{aligned}
$$


with

$$
\begin{aligned}
\alpha_{1} & =\pi\left(2 e_{0}+e_{1}\right), \\
\alpha_{2} & =\frac{\pi}{8}\left(-16 e_{0}-14 e_{1}+5 e_{3}+5 e_{5}+e_{7}+e_{10}\right), \\
\alpha_{3} & =-e_{2}-e_{4}+e_{6}, \\
\alpha_{4} & =-3 e_{2}+7 e_{4}-2 e_{6}, \\
\alpha_{5} & =16 e_{2}+6 e_{4}+4 e_{6}, \\
\alpha_{6} & =e_{9}-e_{12}+e_{13}, \\
\alpha_{7} & =2 e_{12}-4 e_{13}, \\
\alpha_{8} & =e_{13} \\
\alpha_{9} & =-e_{8}+e_{11}, \\
\alpha_{10} & =3 e_{8}-13 e_{11}, \\
\bar{g}_{1}\left(r_{0}\right) & =E\left(2 r_{0}^{4}\right)+\sqrt{1-2 r_{0}^{4}} E\left(1-1 /\left(1-2 r_{0}^{4}\right)\right), \\
\bar{g}_{2}\left(r_{0}\right) & =\left(1-2 r_{0}^{4}\right) K\left(2 r_{0}^{4}\right)+\sqrt{1-2 r_{0}^{4}} K\left(1-1 /\left(1-2 r_{0}^{4}\right)\right), \\
\bar{g}_{3}\left(r_{0}\right) & =\sqrt{1-2 r_{0}^{4}}-1 .
\end{aligned}
$$

Using the expression of each $\alpha_{i}$ one can easily check that $\alpha_{1}, \alpha_{2}, \ldots, \alpha_{10}$ are independent constants.

To simplify the computation, we let $s=\sqrt{1-2 r_{0}^{4}}, s \in(0,1)$. By using the definition of the elliptic functions, we have

$$
\begin{aligned}
s E\left(1-1 / s^{2}\right)+E\left(1-s^{2}\right) & =2 E\left(1-s^{2}\right), \\
s K\left(1-1 / s^{2}\right)+s^{2} K\left(1-s^{2}\right) & =2 s^{2} K\left(1-s^{2}\right) .
\end{aligned}
$$

Hence we obtain

$$
240 r_{0}^{7} \mathcal{F}\left(r_{0}\right)=G(s)=b_{0} f_{0}(s)+b_{1} f_{1}(s)+\cdots+b_{7} f_{7}(s), s \in(0,1),
$$

where $f_{i}(s), i=0,1, \ldots, 7$, are the functions defined in (10), and the constants $b_{0}, b_{1}, \ldots, b_{7}$ in (9) are independent constants each of which is a linear combination of $\alpha_{1}, \alpha_{2}, \ldots, \alpha_{10}$.

By Theorem 4.3 of Appendix 1, the lemma is proved.

Next, denoted by $W_{i}(s)$ the Wronskian determinant for the functions $f_{0}, f_{1}, \ldots, f_{i}$ depending on $s$ :

$$
W_{i}(s)=W\left(f_{0}, \ldots, f_{i}\right)(s), i=0,1, \ldots, 7 .
$$

In what follows we will show that all the Wronskian determinants have not zeros except $W_{6}$.

By direct calculation we obtain

$$
\begin{aligned}
& W_{0}(s)=(1-s)^{2}, \\
& W_{1}(s)=(1-s)^{4} \\
& W_{2}(s)=2(1-s)^{6}, \\
& W_{3}(s)=-12(1-s)^{8}, \\
& W_{4}(s)=288(1-s)^{10} \\
& W_{5}(s)=Y_{5}(s)\left(Z_{50}(s) g_{2}(s)+Z_{51}(s) g_{1}(s)\right), \\
& W_{6}(s)=Y_{6}(s)\left(Z_{60}(s) g_{2}^{2}(s)+Z_{61}(s) g_{2}(s) g_{1}(s)+Z_{62}(s) g_{1}^{2}(s)\right), \\
& W_{7}(s)=Y_{7}(s)\left(Z_{70}(s) g_{2}^{3}(s)+Z_{71}(s) g_{2}^{2}(s) g_{1}(s)+Z_{72}(s) g_{2}(s) g_{1}^{2}(s)+Z_{73}(s) g_{1}^{3}(s)\right),
\end{aligned}
$$


where

$$
\begin{aligned}
& Y_{5}(s)=288 s^{-3}(1-s)^{\frac{15}{2}}(1+s)^{-\frac{5}{2}}, \\
& Z_{50}(s)=1-20 s-33 s^{2}-20 s^{3}+s^{4} \text {, } \\
& Z_{51}(s)=-2\left(1-5 s+10 s^{2}+5 s^{3}+10 s^{4}-5 s^{5}+s^{6}\right) \text {, } \\
& Y_{6}(s)=432 s^{-6}(1-s)^{2}(1+s)^{-8}, \\
& Z_{60}(s)=\sqrt{1-s^{2}}\left(-330-761 s+3720 s^{2}+25036 s^{3}+63490 s^{4}+100713 s^{5}\right. \\
& \left.+102410 s^{6}+66145 s^{7}+23800 s^{8}+3760 s^{9}-770 s^{10}-269 s^{11}\right) \\
& -2\left(210+637 s-2490 s^{2}-22210 s^{3}-67910 s^{4}-129477 s^{5}-160950 s^{6}\right. \\
& \left.-135498 s^{7}-74890 s^{8}-25715 s^{9}-2910 s^{10}+728 s^{11}+240 s^{12}\right), \\
& Z_{61}(s)=\sqrt{1-s^{2}}\left(660+1544 s-2430 s^{2}-6587 s^{3}+18350 s^{4}+65033 s^{5}+107880 s^{6}\right. \\
& \left.+106430 s^{7}+64350 s^{8}+11893 s^{9}-4790 s^{10}-2177 s^{11}+1580 s^{12}+536 s^{13}\right) \\
& +4\left(210+644 s-150 s^{2}-2133 s^{3}+1670 s^{4}+9718 s^{5}+23630 s^{6}+29805 s^{7}\right. \\
& \left.+27970 s^{8}+14822 s^{9}+3430 s^{10}-2877 s^{11}-300 s^{12}+736 s^{13}+240 s^{14}\right), \\
& Z_{62}(s)=s \sqrt{1-s^{2}}\left(-44-1410 s+405 s^{2}+6880 s^{3}-6789 s^{4}-63230 s^{5}-111946 s^{6}\right. \\
& \left.-111330 s^{7}-57735 s^{8}-6880 s^{9}+7555 s^{10}-270 s^{11}-1146 s^{12}-80 s^{13}+4 s^{14}\right) \\
& -2 s\left(28+1590 s+1873 s^{2}-8750 s^{3}-4018 s^{4}+81350 s^{5}+209051 s^{6}+273750 s^{7}\right. \\
& \left.+208594 s^{8}+80650 s^{9}-4307 s^{10}-8050 s^{11}+1712 s^{12}+1560 s^{13}+32 s^{14}\right), \\
& Y_{7}(s)=-3888 s^{-9}(1-s)^{-\frac{7}{2}}(1+s)^{-\frac{27}{2}} \text {, } \\
& Z_{70}(s)=\sqrt{1-s^{2}}\left(12600+126840 s+317850 s^{2}+52804 s^{3}-990100 s^{4}-1480411 s^{5}\right. \\
& +1111220 s^{6}+6912788 s^{7}+11963620 s^{8}+12059608 s^{9}+7333520 s^{10} \\
& +1499322 s^{11}-1510040 s^{12}-1134068 s^{13}+73250 s^{14}+339916 s^{15}+124820 s^{16} \\
& \left.+15361 s^{17}+60 s^{18}\right)-8 \sqrt{1-s^{2}}\left(1-s^{2}\right)^{3} s^{9}(1+s)^{10}\left(3150+31710 s+95130 s^{2}\right. \\
& +77561 s^{3}-209720 s^{4}-618425 s^{5}-351890 s^{6}+1120612 s^{7}+3107560 s^{8} \\
& +4115235 s^{9}+3350990 s^{10}+1407368 s^{11}-195310 s^{12}-611035 s^{13}-252580 s^{14} \\
& \left.+65059 s^{15}+103320 s^{16}+36240 s^{17}+3600 s^{18}\right) \text {, } \\
& Z_{71}(s)=3 \sqrt{1-s^{2}}\left(-8400-85120 s-232660 s^{2}-173008 s^{3}+437450 s^{4}+811463 s^{5}\right. \\
& -2067190 s^{6}-10578234 s^{7}-22263600 s^{8}-30680904 s^{9}-30782260 s^{10} \\
& -22474150 s^{11}-10890340 s^{12}-2297236 s^{13}+798740 s^{14}+530744 s^{15} \\
& \left.-174630 s^{16}-249833 s^{17}-82630 s^{18}-10282 s^{19}-80 s^{20}\right)-24(2100+21280 s \\
& +69900 s^{2}+98437 s^{3}-32560 s^{4}-271689 s^{5}+257650 s^{6}+2845680 s^{7} \\
& +7627330 s^{8}+12561491 s^{9}+14784900 s^{10}+12847024 s^{11}+8032670 s^{12} \\
& +3146115 s^{13}+365000 s^{14}-280461 s^{15}-58190 s^{16}+94628 s^{17}+76050 s^{18} \\
& \left.+24320 s^{19}+2400 s^{20}\right) \text {, } \\
& Z_{72}(s)=3 s \sqrt{1-s^{2}}\left(1120+43540 s+298396 s^{2}+428670 s^{3}-487118 s^{4}-1615600 s^{5}\right. \\
& +1598463 s^{6}+14431800 s^{7}+34113716 s^{8}+48793180 s^{9}+48574944 s^{10} \\
& +34190000 s^{11}+15087426 s^{12}+1926380 s^{13}-1797616 s^{14}-499770 s^{15} \\
& \left.+470882 s^{16}+285160 s^{17}+48663 s^{18}-1040 s^{19}+84 s^{20}+80 s^{21}\right) \\
& +24 s\left(280+13530 s+98799 s^{2}+200770 s^{3}-31141 s^{4}-611730 s^{5}+35248 s^{6}\right. \\
& +4717630 s^{7}+13875311 s^{8}+23495150 s^{9}+27804240 s^{10}+24063400 s^{11} \\
& +14574499 s^{12}+5157020 s^{13}+149657 s^{14}-658770 s^{15}-66644 s^{16} \\
& \left.+208280 s^{17}+108756 s^{18}+14970 s^{19}+320 s^{20}\right) \text {, } \\
& Z_{73}(s)=s^{2} \sqrt{1-s^{2}}\left(480-10712 s-177820 s^{2}-762730 s^{3}-87610 s^{4}+2806841 s^{5}\right. \\
& +909430 s^{6}-14409046 s^{7}-40794440 s^{8}-62886616 s^{9}-63914340 s^{10} \\
& -42197910 s^{11}-14285100 s^{12}+1389476 s^{13}+2447740 s^{14}-233714 s^{15} \\
& \left.-691850 s^{16}-196931 s^{17}+7070 s^{18}+1990 s^{19}-760 s^{20}-8 s^{21}\right) \\
& -8 s^{2}\left(270+538 s+49880 s^{2}+295952 s^{3}+320270 s^{4}-754291 s^{5}\right. \\
& -1276480 s^{6}+4132165 s^{7}+18820380 s^{8}+36641394 s^{9}+45116550 s^{10} \\
& +37467621 s^{11}+19916970 s^{12}+4809620 s^{13}-1224770 s^{14}-907079 s^{15} \\
& \left.+291730 s^{16}+324433 s^{17}+58270 s^{18}+1472 s^{19}+180 s^{20}\right) \text {. }
\end{aligned}
$$


Lemma 3.2. Let $_{g_{1}}$ and $g_{2}$ be the two functions defined in (11) and let $h(s)=g_{1}(s) / g_{2}(s)$. Then $h(s)>0, h^{\prime}(s)<0, s \in(0,1)$ and

$$
\lim _{s \rightarrow 0^{+}} h(s)=+\infty, \lim _{s \rightarrow 1^{-}} h(s)=1, \lim _{s \rightarrow 0^{+}} h^{\prime}(s)=-\infty, \lim _{s \rightarrow 1^{-}} h^{\prime}(s)=-1 .
$$

Proof. It follows directly from the definition of the elliptic integral that $g_{i}(s)>0(i=$ $1,2), s \in(0,1)$ and hence $h(s)>0, s \in(0,1)$. A direct computation shows that

$$
\begin{aligned}
& g_{1}(s)=1-\frac{1}{2} s^{2} \log s+\frac{1}{4} s^{2}(4 \log 2-1)+o\left(s^{2}\right), \\
& g_{2}(s)=-s^{2} \log s+2 s^{2} \log 2+o\left(s^{2}\right),
\end{aligned}
$$

where $s \rightarrow 0^{+}$. Thus the first and the third equalities of (15) hold.

Similarly, we find that

$$
\begin{aligned}
& g_{1}(s)=\frac{\pi}{2}\left(1-\frac{1}{2}(1-s)+\frac{1}{16}(1-s)^{2}+O\left((1-s)^{3}\right)\right) \\
& g_{2}(s)=\frac{\pi}{2}\left(1-\frac{3}{2}(1-s)+\frac{5}{16}(1-s)^{2}+O\left((1-s)^{3}\right)\right)
\end{aligned}
$$

as $s \rightarrow 1^{-}$. This verifies the second and the fourth equalities of (15).

Next we go to prove that $h^{\prime}(s)<0, s \in(0,1)$. By straightforward calculation we find $d h / d s=\left(1-2 h+h^{2} s^{2}\right) /\left(s-s^{3}\right)$. Hence $h(s)$ is a solution of system

$$
\dot{h}=s^{2} h^{2}-2 h+1, \quad \dot{s}=s-s^{3} .
$$

System (17) has two invariant straight lines $s=0$ and $s=1$ as well as two singularities at $S_{1}(0,1 / 2)$ and $S_{2}(1,1)$, where $S_{1}$ is a saddle and $S_{2}$ is a saddle-node of system (17). Moreover, system (17) has two horizontal isocline curves

$$
h_{+}(s)=\frac{1}{1-\sqrt{1-s^{2}}} \text { and } h_{-}(s)=\frac{1}{1+\sqrt{1-s^{2}}},
$$

satisfying

$$
h_{+}^{\prime}(s)<0, h_{-}^{\prime}(s)>0, h_{+}(0)=+\infty, h_{-}(0)=1 / 2, h_{+}(1)=h_{-}(1)=1 .
$$

Obviously,

$$
h^{\prime}(s)=s^{2}\left(h(s)-h_{+}(s)\right)\left(h(s)-h_{-}(s)\right) /\left(s-s^{3}\right) .
$$

In view of (16) and (18), it follows that

$$
h_{-}(s)<h(s)<h_{+}(s), s \rightarrow 1^{-} .
$$

We assert that

$$
h_{-}(s)<h(s)<h_{+}(s), s \in(0,1) .
$$

Indeed, if there exists some point $s_{0} \in(0,1)$ such that $h\left(s_{0}\right) \geq h_{+}\left(s_{0}\right)$, then by (19) we find $h^{\prime}\left(s_{0}\right) \geq 0$. By the monotonicity of $h_{+}(s)$ we know that $h(s)>h_{+}(s)$ for all $s_{0}<s<1$. This contradicts (20). Hence $h(s)<h_{+}(s)$ for $s \in(0,1)$. If there exists some point $s_{0} \in(0,1)$ such that $h\left(s_{0}\right)=h_{-}\left(s_{0}\right)$, then by (19) we know that $h^{\prime}\left(s_{0}\right)=0$. Since $h_{-}^{\prime}\left(s_{0}\right)>0$, it follows $h(s)<h_{-}(s)$ for $s \rightarrow s_{0}^{+}$. Using this fact we find that the curve $h=h(s)$ cannot go across the curve $h=h_{-}(s)$ at any point $s_{1}>s_{0}$ because once $h\left(s_{1}\right)=h_{-}\left(s_{1}\right)$, then it must hold that $h(s)<h_{-}(s)$ for $s \rightarrow s_{1}^{+}$. This also contradicts (20). Hence $h(s)>h_{-}(s), s \in(0,1)$.

Finally, combining (21) and (19) we conclude that $d h / d s<0, s \in(0,1)$.

Lemma 3.3. The function $W_{5}(s)$ does not vanish in the open interval $(0,1)$. 
Proof. By using Sturm's Theorem (see [18]) and $Z_{51}(0)=-2$, we find that $Z_{51}(s)<0$ for all $s \in(0,1)$. Hence we have

$$
W_{5}(s)=Y_{5}(s) Z_{51}(s) g_{2}(s)\left(\frac{Z_{50}(s)}{Z_{51}(s)}+\frac{g_{1}(s)}{g_{2}(s)}\right), s \in(0,1)
$$

A direct computation leads to $Z_{50}\left(s_{0}\right)=0$, where

$$
s_{0}=5+3 \sqrt{15} / 2-\sqrt{231+60 \sqrt{15}} / 2 \approx 0.0463551 .
$$

Again, by Sturm's Theorem we find that $Z_{50}(s)>0$ for $s \in\left(0, s_{0}\right)$ and $Z_{50}(s)<0$ for $s \in\left(s_{0}, 1\right)$. Further,

$$
\frac{d}{d s}\left(\frac{Z_{50}(s)}{Z_{51}(s)}\right)=\frac{2 p_{9}(s)}{Z_{51}^{2}(s)}
$$

where $p_{9}(s)=15+86 s-290 s^{2}-364 s^{3}-575 s^{4}-274 s^{5}+190 s^{6}+68 s^{7}-65 s^{8}+2 s^{9}$. Using Sturm's Theorem we get that $p_{9}(s)>0, s \in(0,1 / 5)$. This fact, being combined with $Z_{50}(0) / Z_{51}(0)=-1 / 2$, yields that

$$
\frac{Z_{50}(s)}{Z_{51}(s)}>0, s \in\left(s_{0}, 1\right) \quad \text { and } \quad \frac{Z_{50}(s)}{Z_{51}(s)} \in\left(-\frac{1}{2}, 0\right), s \in\left(0, s_{0}\right)
$$

Since by Lemma 3.2 we have $g_{1}(s) / g_{2}(s)>1$, it follows from $(22)$ and $(23)$ that $W_{5}(s) \neq 0$ for all $s \in(0,1)$.

Next, we will determine the sign of the functions $W_{6}(s)$ and $W_{7}(s)$. In order to make the computation easier we need to make the transformation of variable $r=\sqrt{(1-s) /(1+s)}$ or equivalently, $s=\left(1-r^{2}\right) /\left(1+r^{2}\right)$. We also need the following lemma. Let

$$
\bar{h}(r)=\left.\frac{g_{1}(s)}{g_{2}(s)}\right|_{s=\left(1-r^{2}\right) /\left(1+r^{2}\right)}, \quad r \in(0,1) .
$$

Lemma 3.4. The function $h=\bar{h}(r)$ is the solution of the differential system

$$
\dot{h}=\left((r-1)^{2} h-r^{2}-1\right)\left((r+1)^{2} h-r^{2}-1\right), \quad \dot{r}=r\left(r^{4}-1\right),
$$

satisfying $\bar{h}^{\prime}(r)>0$ for $r \in(0,1), \bar{h}(0)=1$, and $\lim _{r \rightarrow 1^{-}} \bar{h}(r)=+\infty$.

Proof. The conclusion follows from the proof of Lemma 3.2 by direct calculation.

Lemma 3.5. The function $W_{6}(s)$ has a unique zero in $(0,1)$ and this zero is simple.

Proof. Let $s=\left(1-r^{2}\right) /\left(1+r^{2}\right)$, for $0<r<1$. Then it follows from the definition of $W_{6}(s)$ that

$$
\bar{W}_{6}(r):=W_{6}\left(\frac{1-r^{2}}{1+r^{2}}\right)=\bar{Y}_{6}(r) \bar{g}_{2}^{2}(r)\left(C_{60}(r)+C_{61}(r) \bar{h}(r)+C_{62}(r) \bar{h}^{2}(r)\right)
$$


where $\bar{h}(r)$ is the function defined in (24) and

$$
\begin{aligned}
\bar{Y}_{6}(r)= & \left.\frac{2 Y_{6}(s)}{\left(1+r^{2}\right)^{16}}\right|_{s=\left(1-r^{2}\right) /\left(1+r^{2}\right)}, \\
\bar{g}_{2}(r)= & \left.g_{2}(s)\right|_{s=\left(1-r^{2}\right) /\left(1+r^{2}\right)^{\prime}} \\
C_{60}(r)= & -\left(1+r^{2}\right)^{4}\left(-620235-386944 r+63082 r^{2}+4352 r^{3}+1114260 r^{4}\right. \\
& +747808 r^{5}-136770 r^{6}-21280 r^{7}+349425 r^{8}+201312 r^{9}-5852 r^{10} \\
& +97568 r^{11}+90000 r^{12}+59232 r^{13}+29692 r^{14}-39392 r^{15}-39225 r^{16} \\
& -6880 r^{17}-10350 r^{18}+18912 r^{19}+24540 r^{20}-1152 r^{21}-1242 r^{22} \\
& \left.+2304 r^{23}+2835 r^{24}\right), \\
C_{61}(r)= & 2\left(1+r^{2}\right)^{2}\left(107415+181136 r-33728 r^{2}+11072 r^{3}-399945 r^{4}-731304 r^{5}\right. \\
& +149536 r^{6}-47384 r^{7}+1468275 r^{8}+1717984 r^{9}-340256 r^{10}+125584 r^{11} \\
& +2454435 r^{12}+1436304 r^{13}-95680 r^{14}+188016 r^{15}+156165 r^{16} \\
& -122704 r^{17}+100096 r^{18}-61184 r^{19}-145275 r^{20}-30376 r^{21}-23136 r^{22} \\
& \left.+33384 r^{23}+39345 r^{24}-1632 r^{25}-2592 r^{26}+4464 r^{27}+5985 r^{28}\right), \\
C_{62}(r)= & \left(1-r^{2}\right)\left(-835065-346016 r-836827 r^{2}-363808 r^{3}+3987923 r^{4}\right. \\
& +1598928 r^{5}+4009041 r^{6}+1699296 r^{7}-8764869 r^{8}-3404464 r^{9} \\
& -8875247 r^{10}-3660192 r^{11}+1910023 r^{12}+319232 r^{13}+2054749 r^{14} \\
& +561472 r^{15}+3501349 r^{16}+1423232 r^{17}+3542143 r^{18}+1373728 r^{19} \\
& -275927 r^{20}+31056 r^{21}-405789 r^{22}-8224 r^{23}+15321 r^{24}+43728 r^{25} \\
& \left.+44043 r^{26}+4512 r^{27}+2493 r^{28}+6624 r^{29}+9135 r^{30}\right) .
\end{aligned}
$$

Define

$$
\bar{w}_{6}(r, h)=C_{60}(r)+C_{61}(r) h+C_{62}(r) h^{2} .
$$

We will show that on the curve $C:=\left\{(r, h) \mid \bar{w}_{6}(r, h)=0, r \in(0,1)\right\}$, there is a unique point $P$ at which vector field $(25)$ is tangent to $C$. We call $P$ the contact point with the vector field (25). In fact, by direct computation we obtain

$$
D(r, h):=\left(\partial \bar{w}_{6} / \partial r, \partial \bar{w}_{6} / \partial h\right) \cdot(\dot{r}, \dot{h})=-2 \sum_{i=0}^{3} d_{i}(r) h^{i}
$$

where

$$
\begin{aligned}
d_{0}(r)= & \left(1+r^{2}\right)^{4}\left(-107415+12336 r+2451586 r^{2}+1530176 r^{3}-4561843 r^{4}\right. \\
& -2896872 r^{5}-3880856 r^{6}-2845432 r^{7}+4366665 r^{8}+2321984 r^{9}-1985574 r^{10} \\
& -1627056 r^{11}-175627 r^{12}-500432 r^{13}-524832 r^{14}+797392 r^{15}+938867 r^{16} \\
& +960688 r^{17}+476566 r^{18}-543968 r^{19}-529425 r^{20}-119176 r^{21}-195912 r^{22} \\
& \left.+200040 r^{23}+275163 r^{24}-24288 r^{25}-27378 r^{26}+31248 r^{27}+39375 r^{28}\right), \\
d_{1}(r)= & -\left(1+r^{2}\right)^{2}\left(-1049895-889424 r-726170 r^{2}-1522240 r^{3}+7709074 r^{4}\right. \\
& +7536696 r^{5}+1889478 r^{6}+6432616 r^{7}-31052618 r^{8}-28741640 r^{9}-6577218 r^{10} \\
& -16058648 r^{11}-6172022 r^{12}+328016 r^{13}-22042946 r^{14}-12811872 r^{15} \\
& +33763160 r^{16}+23233568 r^{17}-4741326 r^{18}+5413264 r^{19}+1388182 r^{20} \\
& -2485928 r^{21}+3589522 r^{22}-1915736 r^{23}-3612822 r^{24}-869704 r^{25}-768726 r^{26} \\
& \left.+678984 r^{27}+822606 r^{28}-77664 r^{29}-113814 r^{30}+122832 r^{31}+170415 r^{32}\right), \\
d_{2}(r)= & -\left(1-r^{4}\right)\left(1777545+1046176 r+3311818 r^{2}+1457408 r^{3}-16459052 r^{4}\right. \\
& -8289520 r^{5}-15874018 r^{6}-7006192 r^{7}+62475516 r^{8}+28826544 r^{9}+35342330 r^{10} \\
& +15708784 r^{11}-49795012 r^{12}-19466016 r^{13}-8698050 r^{14}-3515776 r^{15} \\
& -24983266 r^{16}-12853376 r^{17}-14258354 r^{18}-5372832 r^{19}+31346828 r^{20} \\
& +11380816 r^{21}+2668682 r^{22}+500624 r^{23}-4087764 r^{24}-691664 r^{25}-471570 r^{26} \\
& \left.+404016 r^{27}+455268 r^{28}-51264 r^{29}-120294 r^{30}+84960 r^{31}+121905 r^{32}\right),
\end{aligned}
$$




$$
\begin{aligned}
d_{3}(r)= & -\left(1-r^{2}\right)^{3}\left(-835065-346016 r-836827 r^{2}-363808 r^{3}+3987923 r^{4}\right. \\
& +1598928 r^{5}+4009041 r^{6}+1699296 r^{7}-8764869 r^{8}-3404464 r^{9}-8875247 r^{10} \\
& -3660192 r^{11}+1910023 r^{12}+319232 r^{13}+2054749 r^{14}+561472 r^{15}+3501349 r^{16} \\
& +1423232 r^{17}+3542143 r^{18}+1373728 r^{19}-275927 r^{20}+31056 r^{21}-405789 r^{22} \\
& -8224 r^{23}+15321 r^{24}+43728 r^{25}+44043 r^{26}+4512 r^{27}+2493 r^{28}+6624 r^{29} \\
& \left.+9135 r^{30}\right) .
\end{aligned}
$$

By using Sturm's Theorem we find $d_{3}(r)>0, r \in(0,1)$. Further, the resultant of $\bar{w}_{6}(r, h)$ and $D(r, h)$ with respect to $h$ is a polynomial in the variable $r$ of degree 166, which can be proved by applying Sturm's Theorem, to has a unique simple zero $r_{0} \in(0,1)$ with $9 / 10<r_{0}<91 / 100$. Hence there exists a unique $h_{0}$ such that

$$
\bar{w}_{6}\left(r_{0}, h_{0}\right)=D\left(r_{0}, h_{0}\right)=0 .
$$

This confirms that on the curve $C$ there is a unique point $\left(r_{0}, h_{0}\right)$ at which the vector field (25) is tangent to $C$.

By direct computation we have $C_{61}^{2}-4 C_{60} C_{62}=3600\left(1+r^{2}\right)^{4} p_{56}(r)$, where $p_{56}(r)$ is a polynomial of degree 56. Again, we can apply Sturm's Theorem to prove that $p_{56}(r)>0$ and $C_{62}(r)<0$ in $r \in(0,1)$. Let

$$
C_{-}=\left\{h=\bar{h}_{-}(r)=\frac{-C_{61}-\sqrt{C_{61}^{2}-4 C_{60} C_{62}}}{2 C_{62}}\right\}
$$

and

$$
C_{+}=\left\{h=\bar{h}_{+}(r)=\frac{-C_{61}+\sqrt{C_{61}^{2}-4 C_{60} C_{62}}}{2 C_{62}}\right\}
$$

be the two branches of the curve $C$ in the $(r, h)$-plane. A calculation shows that

$$
\bar{h}_{-}(0)=1, \bar{h}_{+}(0)=-\frac{179}{241}, \bar{h}(0)=1, \bar{h}_{-}^{\prime}(0)=\frac{64}{231}, \bar{h}_{+}^{\prime}(0)=\frac{96160}{1916673}, \bar{h}^{\prime}(0)=0,
$$

$\bar{h}_{+}(1)=1 / 2$ and when $r \rightarrow 1^{-}$,

$$
\bar{h}_{-}(r)=\frac{15}{1-r}+\cdots, \quad \bar{h}(r)=\frac{1}{(\log 4-\log (1-r))(r-1)^{2}}+\cdots,
$$

where the dots denote the terms which are infinitesimal being compared to the former one.

It follows that

$$
\bar{h}_{+}(r)<\bar{h}(r)<\bar{h}_{-}(r), \text { as } r \rightarrow 0^{+}, \bar{h}_{+}(r)<\bar{h}_{-}(r)<\bar{h}(r), \text { as } r \rightarrow 1^{-} .
$$

Obviously, the curve $\Gamma=\{h=\bar{h}(r)\}$ intersects $C_{-}$in at least one point $\left(r^{*}, h^{*}\right)$. By an observation on the direction of vector field (25) at the two endpoints of the segment of curve $\left\{(r, h) \mid h=\bar{h}_{-}(r), r \in\left(0, r^{*}\right]\right\}$, we find that there exists a point $P$ at which the vector field (25) is tangent to the curve $C_{-}$(see Figure 2). Since the contact point $P$ is unique, the curve $\Gamma$ cannot intersect $C_{-}$in other point. On the other hand, the curve $\Gamma$ has not common point with $C_{+}$, otherwise a second contact point will emerge. Therefore the function $\bar{w}_{6}(r, \bar{h}(r))$ has a unique zero in the interval $(0,1)$. This yield that $\bar{W}_{6}(r)$ has a unique zero in the interval $(0,1)$.

Finally, since $r_{0}<91 / 100$ and $\bar{h}(91 / 100) \approx 30.54045135<h_{-}(91 / 100) \approx 35.81140037$, it follows that $r_{0}<r^{*}$. This means that $\left(r^{*}, h^{*}\right)$ is not the contact point of $C_{-}$with the vector field. Therefore, the unique zero of $\bar{W}_{6}(r)$ is simple and thus the required conclusion holds.

Lemma 3.6. The function $W_{7}(s)$ does not vanish in the open interval $(0,1)$.

Proof. By taking transformation $s=\left(1-r^{2}\right) /\left(1+r^{2}\right), 0<r<1$, we obtain from

$$
W_{7}(s)=Y_{7}(s) g_{2}^{3}(s)\left(Z_{70}(s)+Z_{71}(s) \frac{g_{1}(s)}{g_{2}(s)}+Z_{72}(s) \frac{g_{1}^{2}(s)}{g_{2}^{2}(s)}+Z_{73}(s) \frac{g_{1}^{3}(s)}{g_{2}^{3}(s)}\right)
$$




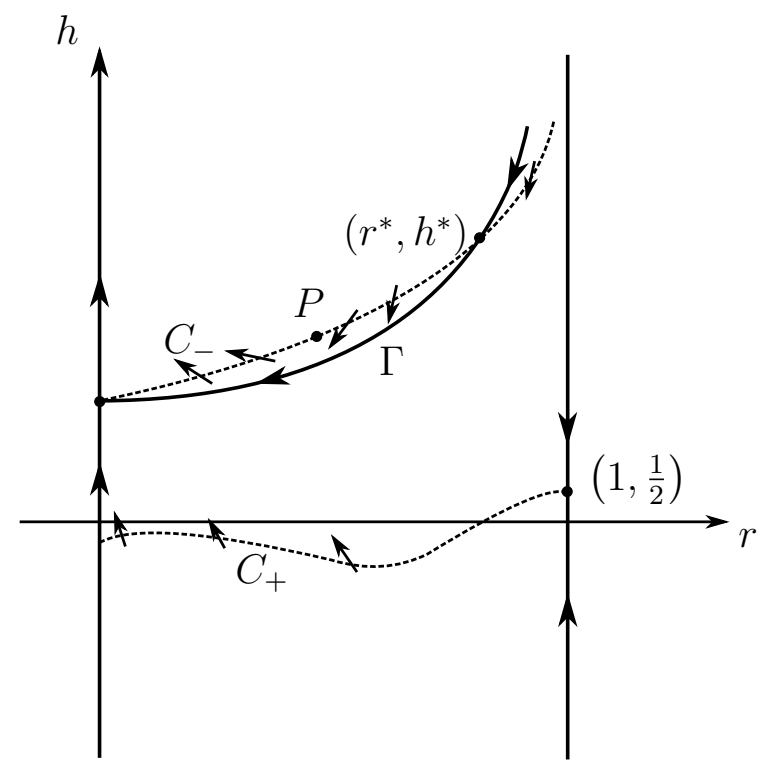

Figure 2. The curve $\Gamma$ has a unique common point with $C_{-}$.

that

$$
\bar{W}_{7}(r):=\left.W_{7}(s)\right|_{s=\left(1-r^{2}\right) /\left(1+r^{2}\right)}=\bar{Y}_{7}(r) \bar{g}_{2}^{3}(r) w_{7}(r)
$$

with

$$
w_{7}(r)=C_{70}(r)+C_{71}(r) \bar{h}(r)+C_{72}(r) \bar{h}^{2}(r)+C_{73}(r) \bar{h}^{3}(r)
$$

where $\bar{h}(r)$ is the function defined in (24) and

$$
\begin{aligned}
\bar{Y}_{7}(r) & =\left.\frac{16 r Y_{7}(s)}{\left(1+r^{2}\right)^{45}}\right|_{s=\left(1-r^{2}\right) /\left(1+r^{2}\right)}, \\
\bar{g}_{2}(r) & =\left.g_{2}(s)\right|_{s=\left(1-r^{2}\right) /\left(1+r^{2}\right)} \\
C_{70}(r) & =4 r\left(2301810+59701740 r^{2}+727558755 r^{4}-364073500959 r^{6}\right. \\
& +3428595727383 r^{8}-10544549722741 r^{10}+3730074158113 r^{12} \\
& +49576965802069 r^{14}-40961684822285 r^{16}-396767446632771 r^{18} \\
& +1609108209115716 r^{20}-3203874112868486 r^{22}+4488939441796380 r^{24} \\
& -4090928490421940 r^{26}+3532364976473268 r^{28}-553179108109916 r^{30} \\
& +1013150621056664 r^{32}+1960313990634764 r^{34}+589939846153370 r^{36} \\
& +1620210743086006 r^{38}+485382645874034 r^{40}+531165876327274 r^{42} \\
& +41364680501774 r^{44}-94823180435898 r^{46}-177772367419966 r^{48} \\
& -169241753304026 r^{50}-122994867094516 r^{52}-75347197109816 r^{54} \\
& -38883154071124 r^{56}-17219939255972 r^{58}-6420781546876 r^{60} \\
& -1958359101836 r^{62}-448781050130 r^{64}-53625628864 r^{66}+12582124635 r^{68} \\
& +10801790529 r^{70}+4279675055 r^{72}+1249653091 r^{74}+292463001 r^{76} \\
& \left.+55639117 r^{78}+8432979 r^{80}+963045 r^{82}+73560 r^{84}+2790 r^{86}\right),
\end{aligned}
$$




$$
\begin{aligned}
& C_{71}(r)=-3\left(1+r^{2}\right)^{23}\left(62214075+32618040 r+57649725 r^{2}+31801560 r^{3}\right. \\
& -311083290 r^{4}-161979460 r^{5}-284045278 r^{6}-156667936 r^{7} \\
& +1118662427 r^{8}+566796672 r^{9}+1015532421 r^{10}+545264968 r^{11} \\
& +1483781736 r^{12}+882038312 r^{13}+1421172920 r^{14}+856116840 r^{15} \\
& +32362070 r^{16}-26403192 r^{17}+175436346 r^{18}+24968536 r^{19} \\
& -2404284 r^{20}-55354400 r^{21}+31425964 r^{22}-122819896 r^{23} \\
& -129246866 r^{24}-82418248 r^{25}-147649550 r^{26}-83415400 r^{27} \\
& -50931800 r^{28}-40785832 r^{29}-66284296 r^{30}-5366728 r^{31} \\
& -2020081 r^{32}-3163552 r^{33}-1758167 r^{34}+617456 r^{35}+624838 r^{36} \\
& \left.+127460 r^{37}+489090 r^{38}+19640 r^{39}+50775 r^{40}+14760 r^{41}+40425 r^{42}\right) \text {, } \\
& C_{72}(r)=3\left(-1+r^{2}\right)\left(1+r^{2}\right)^{21}\left(-113149575-48972840 r-219419550 r^{2}\right. \\
& -96894000 r^{3}+524903715 r^{4}+226248140 r^{5}+1216381628 r^{6} \\
& +542101276 r^{7}-1447999469 r^{8}-628726896 r^{9}-3888634006 r^{10} \\
& -1779992536 r^{11}-2876628303 r^{12}-1259091264 r^{13}-2141852592 r^{14} \\
& -754743200 r^{15}+1112715114 r^{16}+583398240 r^{17}+4296755236 r^{18} \\
& +1852262832 r^{19}+2357760878 r^{20}+907216248 r^{21}+687064168 r^{22} \\
& +234254008 r^{23}+208154838 r^{24}+151069392 r^{25}-302393084 r^{26} \\
& -153692000 r^{27}-210572686 r^{28}-204720480 r^{29}-215766192 r^{30} \\
& -104223744 r^{31}-131334803 r^{32}-41765176 r^{33}-20357126 r^{34} \\
& -11754496 r^{35}-11547809 r^{36}+729436 r^{37}+904508 r^{38}+33740 r^{39} \\
& \left.+444615 r^{40}+42400 r^{41}+114450 r^{42}+18360 r^{43}+50925 r^{44}\right), \\
& C_{73}(r)=-\left(-1+r^{2}\right)^{2}\left(1+r^{2}\right)^{20}\left(164085075+58271640 r+318975300 r^{2}\right. \\
& +115256400 r^{3}-920074365 r^{4}-320089980 r^{5}-2079306888 r^{6} \\
& -746616756 r^{7}+2728744989 r^{8}+941220096 r^{9}+7172532884 r^{10} \\
& +2616432392 r^{11}+779348149 r^{12}+546885328 r^{13}-4995512160 r^{14} \\
& -1385204368 r^{15}-4685978914 r^{16}-1569573552 r^{17}-4525683128 r^{18} \\
& -2222726784 r^{19}+166924638 r^{20}-650651016 r^{21}+4249280720 r^{22} \\
& +1445886696 r^{23}+2224407802 r^{24}+1065394144 r^{25}+814165128 r^{26} \\
& +244092272 r^{27}+320088074 r^{28}-95701872 r^{29}-346329440 r^{30} \\
& -166958928 r^{31}-180571809 r^{32}-99999912 r^{33}-42394764 r^{34} \\
& -27896336 r^{35}-30911889 r^{36}+462756 r^{37}+365688 r^{38}-212820 r^{39} \\
& \left.+107865 r^{40}+50400 r^{41}+137700 r^{42}+21960 r^{43}+61425 r^{44}\right) \text {. }
\end{aligned}
$$

The number of zeros of $\bar{W}_{7}(r)$ in $(0,1)$ equals the number of intersection points of the curve $C=\left\{C_{70}(r)+C_{71}(r) h+C_{72}(r) h^{2}+C_{73}(r) h^{3}=0\right\}$ with the curve $\Gamma=\{h=\bar{h}(r)\}$ in the $(r, h)$-plane. In what follows we will study the relative positions of $C$ and $\Gamma$. To this end, since $\Gamma$ is not an algebraic curve, we need to establish another auxiliary algebraic curve which is easier for computation.

First, by using Sturm's Theorem we find that $C_{73}(r) \neq 0, r \in(0,1)$. This means that for each fixed $r \in(0,1)$,

$$
\bar{w}_{7}(r, h):=C_{70}(r)+C_{71}(r) h+C_{72}(r) h^{2}+C_{73}(r) h^{3}
$$

is a cubic polynomial of $h$. Let

$$
A=C_{72}^{2}-3 C_{71} C_{73}, B=C_{71} C_{72}-9 C_{70} C_{73}, C=C_{71}^{2}-3 C_{70} C_{72},
$$

and let $\Delta=B^{2}-4 A C$. It is not hard to see that $\Delta$ has exactly two zeros $r_{1}, r_{2}$ in $(0,1)$ with $39 / 50<r_{1}<79 / 100,91 / 100<r_{2}<23 / 25$ and if $r \in\left(0, r_{1}\right) \cup\left(r_{2}, 1\right)$ then $\Delta>0$; if $r \in\left(r_{1}, r_{2}\right)$ then $\Delta<0$. Therefore, the curve $C$ has three branches $C_{1}$ (the lower branch), $C_{2}$ (the middle branch) and $C_{3}$ (the upper branch) with the property that $C_{2}$ and $C_{3}$ have the same endpoints $E_{1}\left(r_{1}, h_{1}\right)$ and $E_{2}\left(r_{2}, h_{2}\right)$. See Figure 3. 


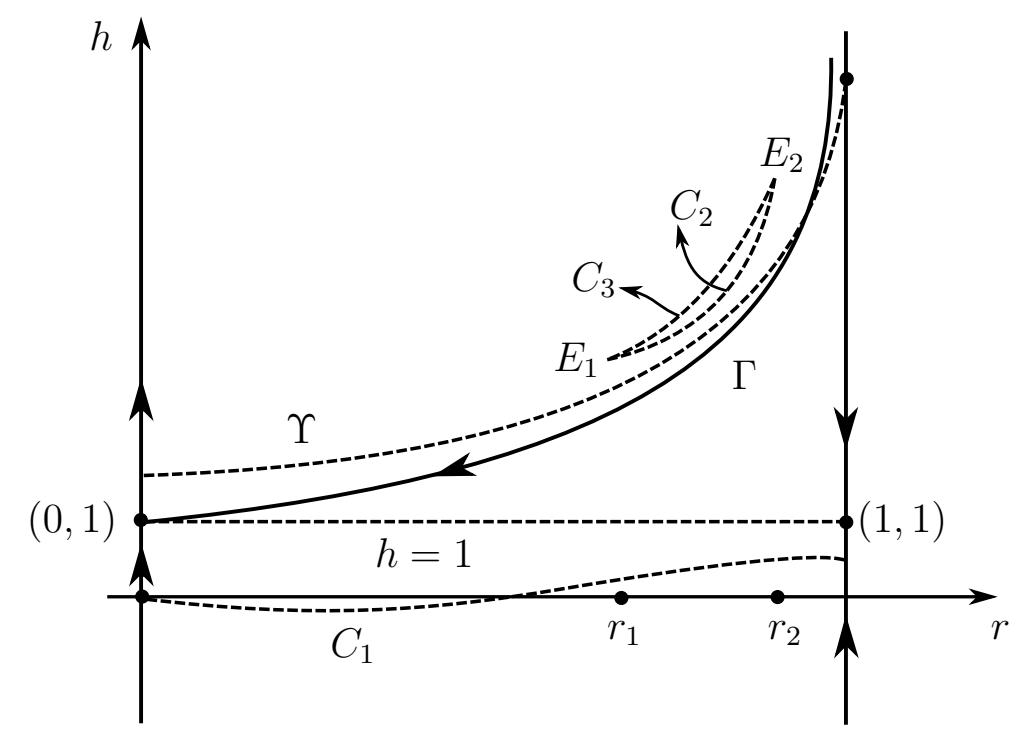

Figure 3. The relative positions of the curve $\Gamma$ and $C$.

Second, we claim that $C_{2} \cup C_{3}$ lies over the curve $\Gamma$. To show this we introduce an auxiliary algebraic curve $\Upsilon=\{h=\Phi(r)\}$ where

$$
\begin{aligned}
\Phi(r)= & \frac{1}{2}\left(5+4 r^{2}+6 r^{4}+8 r^{6}+10 r^{8}+12 r^{10}+13 r^{12}+15 r^{14}+16 r^{16}\right. \\
& +18 r^{18}+20 r^{20}+21 r^{22}+22 r^{24}+38 r^{26}+25 r^{28}+26 r^{30}+28 r^{32}+30 r^{34} \\
& \left.+30 r^{36}+32 r^{38}+34 r^{40}+34 r^{42}+36 r^{44}+38 r^{46}+38 r^{48}+40 r^{50}\right),
\end{aligned}
$$

where $r \in(0,1)$. By direct computations as well as by applying Sturm's Theorem we obtain $\bar{w}_{7}(r, \Phi(r))=p_{238}(r)<0$, where $p_{238}(r)$ is a polynomial of degree 238 . Thus the curve $C$ does not intersect $\Upsilon$. Moreover, in view of that the straight line $r=9 / 10$ intersects the curve $C$ and $\Upsilon$ respectively at the points $\left(9 / 10, c_{1}^{*}\right),\left(9 / 10, c_{2}^{*}\right),\left(9 / 10, c_{3}^{*}\right) \in C$ and $\left(9 / 10, \phi_{1}^{*}\right) \in \Upsilon$, where

$$
c_{1}^{*} \approx 0.1592878, c_{2}^{*} \approx 30.7373179, c_{3}^{*} \approx 40.3056908, \phi_{1}^{*} \approx 26.9337561,
$$

we conclude that $C_{2} \cup C_{3}$ lies over the curve $\Upsilon$, and $C_{1}$ lies below the curve $\Upsilon$. See Figure 3 .

On the other hand, using (25) we obtain by computation that

$$
\begin{aligned}
(\dot{h} & \left.-\Phi^{\prime}(r) \dot{r}\right)\left.\right|_{h=\Phi(r)}=\frac{1}{4}\left(-9+42 r^{2}+3 r^{4}-4 r^{8}-8 r^{10}+18 r^{12}-2 r^{14}+14 r^{16}\right. \\
& -6 r^{18}-36 r^{20}+10 r^{22}+43 r^{24}-826 r^{26}+280 r^{28}+748 r^{30}-23 r^{32}-82 r^{34} \\
& +70 r^{36}+62 r^{38}-132 r^{40}+102 r^{42}+9 r^{44}-124 r^{46}+103 r^{48}+42 r^{50}+4248 r^{52} \\
& +4446 r^{54}-81 r^{56}+82 r^{58}+115 r^{60}+184 r^{62}-20 r^{64}+124 r^{66}+108 r^{68}+72 r^{70} \\
& +128 r^{72}+116 r^{74}+1308 r^{78}-1056 r^{80}+64 r^{82}+136 r^{84}+152 r^{86}-12 r^{88} \\
& \left.+144 r^{90}+156 r^{92}-8 r^{94}+152 r^{96}+160 r^{98}-4 r^{100}+160 r^{102}-1600 r^{104}\right),
\end{aligned}
$$

which has a unique zero in the interval $r_{0} \in(0,1)$ with $0<r_{0}<1 / 2$. Therefore, there exists a unique contact point on the curve $\Upsilon$ with the vector field (25). Taking this into account and noting the fact that

$$
\Phi(0)-\bar{h}(0)>0, \Phi(1 / 2)-\bar{h}(1 / 2)>1>0, \Phi(23 / 25)-\bar{h}(23 / 25)>2 / 5>0,
$$

it is clear that the curve $\left.\Upsilon\right|_{r \in(0,23 / 25)}$ lies over $\left.\Gamma\right|_{r \in(0,23 / 25)}$, otherwise there will exist at least two contact points on $\left.\Upsilon\right|_{r \in(0,23 / 25)}$ with the vector field (25), which leads to a contradiction. 
In summary, according to the relative positions of $\Upsilon$ and $\Gamma$ as well as the relative positions of $\Upsilon$ and $C_{2} \cup C_{3}$, we find that the claim is true.

Third, we claim that $C_{1}$ lies below the curve $\Gamma$. This claim is easy to confirm due to the following facts

(1) $\Gamma$ lies over the straight line $h=1$ (by Lemma 3.4);

(2) $C_{1}$ does not intersect the line $h(r)=1$ because $C_{70}(r)+C_{71}(r)+C_{72}(r)+C_{73}(r) \neq$ $0, r \in(0,1)$ (by applying Sturm's Theorem);

(3) $C_{1}$ is a continuous curve passing through the point $(0,0)$ (because $C_{70}(0)+C_{71}(0) h+$ $C_{72}(0) h^{2}+C_{73}(0) h^{3}=0$ implies $\left.h=0\right)$.

Finally, taking into account the above results, we conclude that the curve $C$ has no common points with the curve $\Gamma$. Thus $\bar{W}_{7}(r) \neq 0$, i.e., $W_{7}(s) \neq 0$.

Proof of statement (b) of Theorem 1.1. It follows from equation (14), Lemma 3.3, Lemma 3.5 and Lemma 3.6 that $w_{i}(s) \neq 0, i=0,1,2,3,4,5,7$ and $w_{6}(s)$ has a simple zero in $(0,1)$. Very recently, in [15] it is proved that if the analytical functions $f_{0}, f_{1}, \ldots, f_{n}: I \rightarrow \mathbb{R}$ satisfy: (1) all the Wronskian determinant $W_{i}(s)=W\left(f_{0}, \ldots, f_{i}\right)(s)$ but $W_{n-1}$ has not zero in the interval $I$, and (2) $W_{n-1}$ has a unique simple zero in $I$, then any linear combination of $f_{0}, f_{1}, \ldots, f_{n}$ can possess at most $n+1$ zeros in $I$, counting with multiplicities. But the authors of [15] do not prove that the upper bound can be reached in the general cases.

In what follows we will show that the upper bound 8 can be reached in our system.

Let $s_{0}=200 / 10001, E_{0}=E\left(1-s_{0}^{2}\right), K_{0}=K\left(1-s_{0}^{2}\right)$ and

$$
k=4224932006353520086857838671137556\left(A E_{0}^{2}+B E_{0} K_{0}+C K_{0}^{2}\right),
$$

where

$$
\begin{aligned}
& A=162632756824646343526934358039550191813769181219039360950399 \\
& B=-26652192151547736499563618692313149392412558977363562257000 \\
& C=5303055781903243156556160927943006794119698485540000000
\end{aligned}
$$

Consider the function

$$
G(s)=a_{0} f_{0}(s)+a_{1} f_{1}(s)+\cdots+a_{6} f_{6}(s)+k f_{7}(s), s \in(0,1) .
$$

By direct calculation we get the power series of $G$ around the point $s_{0}$ :

$$
G(s)=e_{0}+e_{1}\left(s-s_{0}\right)+\cdots+e_{6}\left(s-s_{0}\right)^{6}+e_{7}\left(s-s_{0}\right)^{7}+\cdots,
$$

where $e_{i}$ is the linear combination of $a_{0}, a_{1}, \ldots, a_{6}$. We solve the equations

$$
e_{0}=0, e_{1}=0, \ldots, e_{6}=0,
$$

and find the values of $a_{0}, a_{1}, \ldots, a_{6}$ which have the form

$$
a_{i}=\sum_{j=0}^{3} q_{i j} E_{0}^{j} K_{0}^{3-j}, i=0,1, \ldots, 6,
$$

where each $q_{i j}$ is an integer which occupies many digits. We will not write down here the explicit expression of $a_{i}$ for the sake of brevity. By the way, we would like to point out that our purpose of choosing such a $k$ in (27) is to make the expression of $a_{i}$ to be relative simple. It turns out that

$$
G(s)=e_{7}\left(s-s_{0}\right)^{7}+O\left(\left(s-s_{0}\right)^{8}\right), s \rightarrow s_{0},
$$

where

$$
e_{7}=-\frac{1000600150020001500060001}{264828047171937480000}\left(A_{0} E_{0}^{3}-A_{1} E_{0}^{2} K_{0}+A_{2} E_{0} K_{0}^{2}-A_{3} K_{0}^{3}\right),
$$


with

$A_{0}=9434365215900096702757086133640555232723441933485420521056595192874214200423786458280868229$,

$A_{1}=1732705885903853693884640417850773768026603157714652167368522666300981344222008726672767200$,

$A_{2}=155016549380589651097830255703697459402307866677949892054356184189754798606490809063800000$,

$A_{3}=30929409946148872377165751603166763557011086585236345727947020937984340166396000000000$,

and

$$
e_{7} \approx-8.7875569 \times 10^{97}
$$

On the other hand, at the endpoint $s=1$ we have

$$
G(s)=B(1-s)+o((1-s)),
$$

where

$$
\begin{aligned}
B= & 12800263824853240746592933248429440000532 \pi \\
& \cdot\left(5792157212337693345948517518844704378216313299609167 E_{0}^{2}\right. \\
& \quad-1020168724968577415929102393676106281813032946881000 E_{0} K_{0} \\
& \left.+203589533638142582450403592018967753470820000000 K_{0}^{2}\right) \\
\approx & 1.6008470 \times 10^{91} .
\end{aligned}
$$

The (28) and (29) means that (i) $G$ has a zero at $s_{0}$ with multiplicity 7 , (ii) there exists an $\varepsilon_{0}$ with $s_{0}<\varepsilon_{0}<1$ such that $G(s)$ is negative in $\left(s_{0}, \varepsilon_{0}\right]$, and (iii) $G(s)$ is positive near the endpoint $s=1$.

Fixed the numbers $a_{0}, a_{1}, \ldots, a_{6}$ and $k$, we consider the function

$$
G_{\varepsilon}(s)=G(s)+\sum_{i=0}^{7} \varepsilon_{i} f_{i}(s), s \in(0,1) .
$$

Noting that $f_{i}$ can be extended analytically to $(0,1]$, there exists an $M>0$ such that

$$
G_{\varepsilon}\left(\varepsilon_{0}\right)<\frac{1}{2} G\left(\varepsilon_{0}\right)<0, \quad G_{\varepsilon}(s)>\frac{1}{2} B(1-s), \text { when } s \rightarrow 1^{-},
$$

for all $\left|\varepsilon_{i}\right|<M, i=0,1, \ldots, 7$. Moreover, near $s_{0}$ we find

$$
\sum_{i=0}^{7} \varepsilon_{i} f_{i}(s)=\mu_{0}+\mu_{1}\left(s-s_{0}\right)+\cdots+\mu_{7}\left(s-s_{0}\right)^{7}+\cdots,
$$

where $\mu_{i}=\mu_{i}\left(\varepsilon_{0}, \varepsilon_{1}, \ldots, \varepsilon_{7}\right)$ is linear combination of $\varepsilon_{0}, \varepsilon_{1}, \ldots, \varepsilon_{7}$. One can directly check that the matrix of the coefficients of $\mu_{0}, \mu_{1}, \ldots, \mu_{7}$ with respect to $\varepsilon_{0}, \varepsilon_{1}, \ldots, \varepsilon_{7}$ has rank 8 , and hence $\mu_{0}, \mu_{1}, \ldots, \mu_{7}$ are independent.

Consequently, since $f_{i}$ is analytic at $s_{0}$ and $G$ has a zero at $s_{0}$ with multiplicity 7 , it follows that there exists some small $\left|\varepsilon_{i}\right| \ll M(i=0,1, \ldots, 7)$ (and hence $\mu_{i}$ is small) such that $G_{\varepsilon}$ has exact 7 simple zeros in a neighborhood of $s_{0}$. In view of (30) $G$ has an extra zero in $\left(\varepsilon_{0}, 1\right)$. According to the result of [15], this zero is simple. That is to say, $G_{\varepsilon}$ has 8 simple zeros.

Finally, by Lemma 3.1, using averaging theory of first order systems (4) have at most 8 limit cycles, and the upper bound can be reached. The proof is finished.

\section{Proof of Statement (c) of Theorem 1.1}

The goal of this section is to investigate the number of limit cycles of system (5) which bifurcate from the period annulus of the isochronous center. Before the statement of our result, we should first recall the concept of hypergeometric function. 
Let $H(a, b, c, z)$ be the ordinary hypergeometric function which is defined for $|z|<1$ by the power series

$$
H(a, b, c, z)=\sum_{k=0}^{\infty} \frac{(a)_{k}(b)_{k}}{(c)_{k}} \frac{z^{k}}{k !}
$$

where

$$
(a)_{k}= \begin{cases}1, & k=0 \\ a(a+1) \cdots(a+k-1), & k>0 .\end{cases}
$$

It is undefined (or infinite) if $c$ equals a non-positive integer. Many of the common mathematical functions can be expressed in terms of the hypergeometric function. For example, $(1-z)^{-a}=H(a, 1,1, z)$ and

$$
H\left(\frac{1}{2}, \frac{1}{2}, 1, m\right)=\frac{2 K(m)}{\pi}, H\left(-\frac{1}{2}, \frac{1}{2}, 1, m\right)=\frac{2 E(m)}{\pi} .
$$

For more information on hypergeometric functions, the reader is refereed to chapter 15 of [1].

Lemma 4.1. The maximum number of limit cycles of system (5) which emerge from the period annulus of center of system $(5)_{\varepsilon=9}$, by using averaging theory of first order, is equal to the maximum number of the simple zeros of the function

$$
G(s)=c_{0} g_{0}(s)+c_{1} g_{1}(s)+\cdots+c_{12} g_{12}(s),
$$

where

$$
\begin{aligned}
g_{0}(s) & =s \\
g_{1}(s) & =s^{2} \\
g_{2}(s) & =s \sqrt{1-s} \\
g_{3}(s) & =s H(1,1 / 2,2, s) \\
g_{4}(s) & =s H(1,5 / 2,4, s) \\
g_{5}(s) & =s H(1,3 / 2,3, s) \\
g_{6}(s) & =s^{4 / 3} H(-2 / 3,1 / 2,1, s), \\
g_{7}(s) & =s^{5 / 3} H(-1 / 3,1 / 2,1, s), \\
g_{8}(s) & =s^{5 / 3} H(-1 / 3,3 / 2,2, s), \\
g_{9}(s) & =s^{5 / 3} H(-1 / 3,5 / 2,3, s), \\
g_{10}(s) & =s^{4 / 3} H(-2 / 3,3 / 2,2, s), \\
g_{11}(s) & =s^{4 / 3}\left(-(1-s) H\left(\frac{1}{3}, \frac{5}{2}, 3, s\right)-2 H\left(-\frac{2}{3}, \frac{5}{2}, 3, s\right)\right), \\
g_{12}(s) & =s^{2 / 3}\left((4-9 s) H\left(\frac{2}{3}, \frac{3}{2}, 3, s\right)-(1-s)(4+33 s) H\left(\frac{2}{3}, \frac{5}{2}, 3, s\right)\right),
\end{aligned}
$$

and $c_{0}, c_{1}, \ldots, c_{12}$ are independent arbitrary constants.

Proof. As usual we take the polar coordinate transformation to change system (5) to

$$
\frac{d r}{d \theta}=R_{0}(\theta, r)+\varepsilon R_{1}(\theta, r)+O\left(\varepsilon^{2}\right)
$$

where $R_{0}(\theta, r)=r^{7} \cos \theta \sin \theta$ and $R_{1}(\theta, r)=R_{11}(\theta, r)+R_{12}(\theta, r)$ with

$$
\begin{aligned}
R_{11}(\theta, r) & =r\left(a_{10} C^{2}+b_{01} S^{2}\right)+r^{3}\left[a_{30} C^{4}+\left(a_{12}+b_{21}\right) C^{2} S^{2}+b_{03} S^{4}\right] \\
& +r^{5}\left[a_{50} C^{6}+\left(a_{32}+b_{41}\right) C^{4} S^{2}+\left(a_{14}+b_{23}\right) C^{2} S^{4}+b_{05} S^{6}\right] \\
& +r^{7}\left[a_{70} c^{8}+\left(a_{10}-b_{01}\right) C^{2} S^{2}+\left(a_{52}+b_{61}\right) C^{6} S^{2}+\left(a_{34}+b_{43}\right) C^{4} S^{4}\right. \\
& \left.+\left(a_{16}+b_{25}\right) C^{2} S^{6}+b_{07} S^{8}\right]+r^{9}\left[\left(a_{30}-b_{21}\right) C^{4} s^{2}+\left(a_{12}-b_{03}\right) C^{2} S^{4}\right] \\
& +r^{11}\left[\left(a_{50}-b_{41}\right) C^{6} S^{2}+\left(a_{32}-b_{23}\right) C^{4} S^{4}+\left(a_{14}-b_{05}\right) C^{2} S^{6}\right] \\
& +r^{13}\left[\left(a_{70}-b_{61}\right) C^{8} S^{2}+\left(a_{52}-b_{43}\right) C^{6} S^{4}+\left(a_{34}-b_{25}\right) C^{4} S^{6}+\left(a_{16}-b_{07}\right) C^{2} S^{8}\right],
\end{aligned}
$$


and $R_{12}(\theta, r)$ is a polynomial of degree 13 in $r$ of the form $\sum_{i, j, k} d_{i, j, k} C^{i} S^{j} r^{k}$ where $i$ or $j$ are odd numbers. As before, here $C=\cos \theta, S=\sin \theta$. We do not write down the explicit expression of $R_{12}(\theta, r)$ because it is too long and, as we will see, it does not play any role in further calculation.

The equation (33) $\varepsilon_{\varepsilon=0}$ has the periodic solutions $r\left(\theta, r_{0}\right)=r_{0}\left(1-3 r_{0}^{6} \sin ^{2} \theta\right)^{-1 / 6}$ satisfying $r_{0}=r\left(0, r_{0}\right)$ for $0<r_{0}<3^{-1 / 6}$. The corresponding variational differential equation

$$
\frac{d M}{d \theta}=\frac{\partial}{\partial r} R_{0}\left(\theta, r\left(\theta, r_{0}\right)\right) M
$$

with $M_{r_{0}}(0)=1$ has the fundamental solution

$$
M_{r_{0}}(\theta)=\left(1-3 r_{0}^{6} \sin ^{2} \theta\right)^{-7 / 6}
$$

Next we go to study the maximum number of zeros of the function

$$
\mathcal{F}\left(r_{0}\right)=\int_{0}^{2 \pi} M_{r_{0}}^{-1}(\theta) R_{1}\left(\theta, r\left(\theta, r_{0}\right)\right) d \theta=r_{0}^{7} \int_{0}^{2 \pi} r^{-7}\left(\theta, r_{0}\right) R_{1}\left(\theta, r\left(\theta, r_{0}\right)\right) d \theta,
$$

when $r_{0} \in\left(0,3^{-1 / 6}\right)$.

One can check directly that

$$
\int_{0}^{2 \pi} r^{-7}\left(\theta, r_{0}\right) R_{12}\left(\theta, r\left(\theta, r_{0}\right)\right) d \theta=0
$$

it turns out that

$$
\mathcal{F}\left(r_{0}\right)=r_{0}^{7} \int_{0}^{2 \pi} r^{-7}\left(\theta, r_{0}\right) R_{11}\left(\theta, r\left(\theta, r_{0}\right)\right) d \theta, \quad r_{0} \in\left(0,3^{-1 / 6}\right) .
$$

Further, taking the transformation $r_{0}=(s / 3)^{1 / 6}$, we have

$$
\overline{\mathcal{F}}(s):=\mathcal{F}\left(r_{0}\right)=\int_{0}^{2 \pi}\left(1-s \sin ^{2} \theta\right)^{7 / 6} R_{11}(\theta, \bar{r}(\theta, s)) d \theta, \quad s \in(0,1),
$$

where $\bar{r}(\theta, s)=3^{-1 / 6}\left(s /\left(1-s \sin ^{2} \theta\right)\right)^{1 / 6}$.

By using an algebraic manipulator, we obtain after a long calculation that

$$
\overline{\mathcal{F}}(s)=\frac{\bar{c}_{0} \bar{f}_{0}(s)+\bar{c}_{1} \bar{f}_{1}(s)+\cdots+\bar{c}_{15} \bar{f}_{15}(s)}{s^{17 / 6}(1-s)^{2 / 3}}
$$

where

$$
\begin{aligned}
& \bar{f}_{0}(s)=s^{3}(1-s)^{2 / 3} \\
& \bar{f}_{1}(s)=s^{4}(1-s)^{2 / 3} \\
& \bar{f}_{2}(s)=s^{3}(1-s)^{7 / 6} \\
& \bar{f}_{3}(s)=s^{2}\left((1-s)^{7 / 6}-(1-s)^{2 / 3}\right), \\
& \bar{f}_{4}(s)=8(1-s)^{7 / 6}+(1-s)^{2 / 3}\left(s^{2}+4 s-8\right), \\
& \bar{f}_{5}(s)=2 s(1-s)^{7 / 6}-(1-s)^{2 / 3}\left(2 s-s^{2}\right), \\
& \bar{f}_{6}(s)=s^{10 / 3}(1-s)^{2 / 3} H\left(-\frac{2}{3}, \frac{1}{2}, 1, s\right), \\
& \bar{f}_{7}(s)=s^{11 / 3}(1-s)^{2 / 3} H\left(-\frac{1}{3}, \frac{1}{2}, 1, s\right), \\
& \bar{f}_{8}(s)=s^{11 / 3}(1-s)^{2 / 3} H\left(-\frac{1}{3}, \frac{3}{2}, 2, s\right), \\
& \bar{f}_{9}(s)=s^{11 / 3}(1-s)^{2 / 3} H\left(-\frac{1}{3}, \frac{5}{2}, 3, s\right), \\
& \bar{f}_{10}(s)=s^{14 / 3}\left((1-s)^{2 / 3} H\left(\frac{2}{3}, \frac{3}{2}, 3, s\right)+H\left(\frac{2}{3}, \frac{3}{2}, 3, \frac{s}{s-1}\right)\right)
\end{aligned}
$$




$$
\begin{aligned}
\bar{f}_{11}(s)= & s^{13 / 3}\left((1-s)^{2 / 3} H\left(\frac{1}{3}, \frac{3}{2}, 3, s\right)+(1-s)^{1 / 3} H\left(\frac{1}{3}, \frac{3}{2}, 3, \frac{s}{s-1}\right)\right), \\
\bar{f}_{12}(s)= & s^{14 / 3}\left(2 H\left(\frac{2}{3}, \frac{3}{2}, 3, \frac{s}{s-1}\right)+(1-s)^{2 / 3} H\left(\frac{2}{3}, \frac{5}{2}, 4, s\right)-H\left(\frac{2}{3}, \frac{5}{2}, 4, \frac{s}{s-1}\right)\right), \\
\bar{f}_{13}(s)= & s^{10 / 3}(1-s)^{2 / 3}\left(9 \Gamma\left(-\frac{2}{3}\right) \Gamma\left(\frac{7}{6}\right) H\left(-\frac{2}{3}, \frac{3}{2},-\frac{1}{6}, 1-s\right)\right. \\
& \left.-10 \sqrt{3} \pi^{3 / 2} H\left(-\frac{2}{3}, \frac{3}{2}, 2, s\right)+18(1-s)^{7 / 6} \Gamma\left(-\frac{7}{6}\right) \Gamma\left(\frac{8}{3}\right) H\left(\frac{1}{2}, \frac{8}{3}, \frac{13}{6}, 1-s\right)\right), \\
\bar{f}_{14}(s)= & s^{10 / 3}(1-s)^{1 / 3}\left(243(1-s)^{1 / 3} \Gamma\left(-\frac{2}{3}\right) \Gamma\left(\frac{7}{6}\right) H\left(-\frac{2}{3}, \frac{5}{2},-\frac{1}{6}, 1-s\right)\right. \\
& -360 \sqrt{3} \pi^{3 / 2}(1-s)^{1 / 3} H\left(-\frac{2}{3}, \frac{5}{2}, 3, s\right)+40 \sqrt{3} \pi^{3 / 2} s H\left(\frac{1}{3}, \frac{3}{2}, 3, \frac{s}{s-1}\right) \\
& +20 \sqrt{3} \pi^{3 / 2} s\left((1-s)^{1 / 3} H\left(\frac{1}{3}, \frac{5}{2}, 4, s\right)-H\left(\frac{1}{3}, \frac{5}{2}, 4, \frac{s}{s-1}\right)\right) \\
& \left.+324(1-s)^{3 / 2} \Gamma\left(-\frac{7}{6}\right) \Gamma\left(\frac{11}{3}\right) H\left(\frac{1}{2}, \frac{11}{3}, \frac{13}{6}, 1-s\right)\right), \\
\bar{f}_{15}(s)= & s^{11 / 3}\left(-240(1-s)^{2 / 3} H\left(-\frac{1}{3}, \frac{7}{2}, 4, s\right)+s\left(16 H\left(\frac{2}{3}, \frac{3}{2}, 3, \frac{s}{s-1}\right)\right.\right. \\
& \left.\left.-16 H\left(\frac{2}{3}, \frac{5}{2}, 4, \frac{s}{s-1}\right)+5(1-s)^{2 / 3} H\left(\frac{2}{3}, \frac{7}{2}, 5, s\right)+5 H\left(\frac{2}{3}, \frac{7}{2}, 5, \frac{s}{s-1}\right)\right)\right) .
\end{aligned}
$$

Here $\Gamma(z)$ is the Gamma function defined by $\Gamma(z)=\int_{0}^{\infty} t^{z-1} \mathrm{e}^{-t} d t$ and $\bar{c}_{i}$, for $i=$ $0,1,2, \ldots, 15$, is the linear combination of $a_{i j}$ and $b_{i j}$. We do not give the explicit expressions of $\bar{c}_{i}(i=0,1,2, \ldots, 15)$ because they are too long. We can check by direct calculation that $\bar{c}_{1}, \bar{c}_{2}, \ldots, \bar{c}_{15}$ are independent.

From (31) we have

$$
\begin{aligned}
& \bar{f}_{3}(s)=s^{3}(1-s)^{2 / 3} \frac{\sqrt{1-s}-1}{s}=-\frac{1}{2} s^{3}(1-s)^{2 / 3} H\left(1, \frac{1}{2}, 2, s\right), \\
& \bar{f}_{4}(s)=-\frac{1}{2} s^{3}(1-s)^{2 / 3} \frac{8 \sqrt{1-s}+s^{2}+4 s-8}{-s^{3} / 2}-\frac{1}{2} s^{3}(1-s)^{2 / 3} H\left(1, \frac{5}{2}, 4, s\right), \\
& \bar{f}_{5}(s)=-\frac{1}{4} s^{3}(1-s)^{2 / 3} \frac{s-2+2 \sqrt{1-s}}{-s^{2} / 4}=-\frac{1}{4} s^{3}(1-s)^{2 / 3} H\left(1, \frac{3}{2}, 3, s\right) .
\end{aligned}
$$

Using Pfaff transformation (see chapter 15 of [1])

$$
(1-z)^{a} H(a, b, c, z)=H(a, c-b, c, z /(z-1)),
$$

as well as Gauss' contiguous relation

$$
\begin{aligned}
\frac{a b z}{c} H(a+1, b+1, c+1, z) & =a(H(a+1, b, c, z)-H(a, b, c, z)) \\
& =b(H(a+1, b, c, z)-H(a, b, c, z)) \\
& =(c-1)(H(a, b, c-1, z)-H(a, b, c, z)) \\
& =\frac{(c-a) H(a-1, b, c, z)+(a-c+b z) H(a, b, c, z)}{1-z} \\
& =\frac{(c-b) H(a, b-1, c, z)+(b-c+a z) H(a, b, c, z)}{1-z} \\
& =z \frac{(c-a)(c-b) H(a, b, c+1, z)+c(a+b-c) H(a, b, c, z)}{c(1-z)}
\end{aligned}
$$

and we obtain that

$$
\begin{aligned}
\bar{f}_{10}(s)= & 2 s^{14 / 3}(1-s)^{2 / 3} H\left(\frac{2}{3}, \frac{3}{2}, 3, s\right) \\
\bar{f}_{11}(s)= & 2 s^{13 / 3}(1-s)^{2 / 3} H\left(\frac{1}{3}, \frac{3}{2}, 3, s\right) \\
\bar{f}_{12}(s)= & 2 s^{14 / 3}(1-s)^{2 / 3} H\left(\frac{2}{3}, \frac{5}{2}, 4, s\right) \\
\bar{f}_{14}(s)= & 3 s^{10 / 3}(1-s)^{2 / 3}\left(81 \Gamma\left(-\frac{2}{3}\right) \Gamma\left(\frac{7}{6}\right) H\left(-\frac{2}{3}, \frac{5}{2},-\frac{1}{6}, 1-s\right)\right. \\
& +40 \sqrt{3} \pi^{3 / 2}\left(-H\left(-\frac{2}{3}, \frac{5}{2}, 3, s\right)-2(1-s) H\left(\frac{1}{3}, \frac{5}{2}, 3, s\right)\right) \\
& \left.+108(1-s)^{7 / 6} \Gamma\left(-\frac{7}{6}\right) \Gamma\left(\frac{11}{3}\right) H\left(\frac{1}{2}, \frac{11}{3}, \frac{13}{6}, 1-s\right)\right), \\
\bar{f}_{15}(s)= & \frac{216}{35} s^{8 / 3}(1-s)^{2 / 3}\left((4-9 s) H\left(\frac{2}{3}, \frac{3}{2}, 3, s\right)-(1-s)(4+33 s) H\left(\frac{2}{3}, \frac{5}{2}, 3, s\right)\right) .
\end{aligned}
$$


Further, applying the formula

$$
\begin{aligned}
H(a, b, c, z)= & \frac{\Gamma(c) \Gamma(c-a-b)}{\Gamma(c-a) \Gamma(c-b)} H(a, b, a+b-c+1,1-z) \\
& +(1-z)^{c-a-b} \frac{\Gamma(c) \Gamma(a+b-c)}{\Gamma(a) \Gamma(b)} H(c-a, c-b, c-a-b+1,1-z),
\end{aligned}
$$

for $|\arg (1-z)|<\pi$, we obtain that

$$
\begin{aligned}
\bar{f}_{13}(s)= & s^{10 / 3}(1-s)^{2 / 3}\left(9 \Gamma\left(-\frac{2}{3}\right) \Gamma\left(\frac{8}{3}\right) \Gamma\left(\frac{1}{2}\right)-10 \sqrt{3} \pi^{3 / 2}\right) H\left(-\frac{2}{3}, \frac{3}{2}, 2, s\right) \\
= & \sqrt{\pi} s^{10 / 3}(1-s)^{2 / 3}\left(9 \Gamma\left(-\frac{2}{3}\right) \Gamma\left(\frac{8}{3}\right)-10 \sqrt{3} \pi\right) H\left(-\frac{2}{3}, \frac{3}{2}, 2, s\right) \\
= & -20 \sqrt{3} \pi^{3 / 2} s^{10 / 3}(1-s)^{2 / 3} H\left(-\frac{2}{3}, \frac{3}{2}, 2, s\right) \\
\bar{f}_{14}(s)= & \frac{3}{2} s^{10 / 3}(1-s)^{2 / 3}\left(\left(81 \Gamma\left(-\frac{2}{3}\right) \Gamma\left(\frac{11}{3}\right) \Gamma\left(\frac{1}{2}\right)-80 \sqrt{3} \pi^{3 / 2}\right) H\left(-\frac{2}{3}, \frac{5}{2}, 3, s\right)\right. \\
& \left.-160 \sqrt{3} \pi^{3 / 2}(1-s) H\left(\frac{1}{3}, \frac{5}{2}, 3, s\right)\right) \\
= & \frac{3 \sqrt{\pi}}{2} s^{10 / 3}(1-s)^{2 / 3}\left(\left(81 \Gamma\left(-\frac{2}{3}\right) \Gamma\left(\frac{11}{3}\right)-80 \sqrt{3} \pi\right) H\left(-\frac{2}{3}, \frac{5}{2}, 3, s\right)\right. \\
& \left.+160 \sqrt{3} \pi(s-1) H\left(\frac{1}{3}, \frac{5}{2}, 3, s\right)\right) \\
= & -240 \sqrt{3} \pi^{3 / 2} s^{10 / 3}(1-s)^{2 / 3}\left((1-s) H\left(\frac{1}{3}, \frac{5}{2}, 3, s\right)+2 H\left(-\frac{2}{3}, \frac{5}{2}, 3, s\right)\right) .
\end{aligned}
$$

By the above equalities, we obtain from (34) that

$$
\overline{\mathcal{F}}(s)=s^{-5 / 6}\left(\tilde{c}_{0} f_{0}(s)+\tilde{c}_{1} f_{1}(s)+\cdots+\tilde{c}_{15} f_{15}(s)\right),
$$

where the functions $f_{i}$ 's modulo a nonzero constant are $\bar{f}_{i} /\left(s^{2}(1-s)^{2 / 3}\right)$ :

$$
\begin{aligned}
f_{0}(s) & =s, \\
f_{1}(s) & =s^{2} \\
f_{2}(s) & =s \sqrt{1-s} \\
f_{3}(s) & =s H\left(1, \frac{1}{2}, 2, s\right), \\
f_{4}(s) & =s H\left(1, \frac{5}{2}, 4, s\right), \\
f_{5}(s) & =s H\left(1, \frac{3}{2}, 3, s\right) \\
f_{6}(s) & =s^{4 / 3} H\left(-\frac{2}{3}, \frac{1}{2}, 1, s\right), \\
f_{7}(s) & =s^{5 / 3} H\left(-\frac{1}{3}, \frac{1}{2}, 1, s\right), \\
f_{8}(s) & =s^{5 / 3} H\left(-\frac{1}{3}, \frac{3}{2}, 2, s\right), \\
f_{9}(s) & =s^{5 / 3} H\left(-\frac{1}{3}, \frac{5}{2}, 3, s\right) \\
f_{10}(s) & =s^{8 / 3} H\left(\frac{2}{3}, \frac{3}{2}, 3, s\right), \\
f_{11}(s) & =s^{7 / 3} H\left(\frac{1}{3}, \frac{3}{2}, 3, s\right), \\
f_{12}(s) & =s^{8 / 3} H\left(\frac{2}{3}, \frac{5}{2}, 4, s\right), \\
f_{13}(s) & =s^{4 / 3} H\left(-\frac{2}{3}, \frac{3}{2}, 2, s\right), \\
f_{14}(s) & =-s^{4 / 3}\left((1-s) H\left(\frac{1}{3}, \frac{5}{2}, 3, s\right)+2 H\left(-\frac{2}{3}, \frac{5}{2}, 3, s\right)\right) \\
f_{15}(s) & =s^{2 / 3}\left((4-9 s) H\left(\frac{2}{3}, \frac{3}{2}, 3, s\right)-(1-s)(4+33 s) H\left(\frac{2}{3}, \frac{5}{2}, 3, s\right)\right), \\
&
\end{aligned}
$$

It is also not hard to see that in $(36), \tilde{c}_{0}, \tilde{c}_{1}, \ldots, \tilde{c}_{15}$ are independent constants.

Further, applying repeatedly Gauss' contiguous relation (35), we find that

$$
f_{10}=12 f_{7}-12 f_{8}, f_{11}=6 f_{6}-6 f_{13}, f_{12}=36 f_{8}-36 f_{9} .
$$

It follows from (36) and (37) that

$$
\mathcal{F}\left(r_{0}\right)=\overline{\mathcal{F}}(s)=s^{-5 / 6} \sum_{i=0}^{12} c_{i} g_{i}(s)=s^{-5 / 6} G(s),
$$

where

$$
g_{0}=f_{0}, g_{1}=f_{1}, \ldots, g_{9}=f_{9}, g_{10}=f_{13}, g_{11}=f_{14}, g_{12}=f_{15}
$$


and $c_{0}, c_{1}, \ldots, c_{12}$ are independent constants.

Finally, by Theorem 4.3 of Appendix 1, we obtain the required conclusion.

Proof of statement (c) of Theorem 1.1. First, we claim that the functions $g_{0}, g_{1}, \ldots, g_{12}$ in Lemma 4.1 are linear independent. To show this, we write

$$
\bar{G}(u)=d_{0} g_{0}\left(u^{3}\right)+d_{1} g_{1}\left(u^{3}\right)+\cdots+d_{12} g_{12}\left(u^{3}\right), u>0 .
$$

Then, near the point $u=0$ we have

$$
\begin{aligned}
\frac{8957952 \bar{G}(u)}{u^{3}}= & 8957952\left(d_{0}+d_{2}+d_{3}+d_{4}+d_{5}\right)+8957952\left(d_{10}-3 d_{11}+d_{6}\right) u \\
& -\left(348364800 d_{12}-8957952\left(d_{7}+d_{8}+d_{9}\right)\right) u^{2} \\
& +1119744\left(8 d_{1}-4 d_{2}+2 d_{3}+5 d_{4}+4 d_{5}\right) u^{3} \\
& \left.-1492992\left(3 d_{10}-11 d_{11}+2 d_{6}\right)\right) u^{4}+82944\left(1400 d_{12}\right. \\
& \left.-3\left(6 d_{7}+9 d_{8}+10 d_{9}\right)\right) u^{5}-559872\left(2 d_{2}-2 d_{3}-7 d_{4}-5 d_{5}\right) u^{6} \\
& -124416\left(5 d_{10}-20 d_{11}+3 d_{6}\right) u^{7} \\
& +6912\left(5390 d_{12}-3\left(18 d_{7}+30 d_{8}+35 d_{9}\right)\right) u^{8} \\
& -139968\left(4 d_{2}-5 d_{3}-7\left(3 d_{4}+2 d_{5}\right)\right) u^{9}-6912\left(35 d_{10}-147 d_{11}\right. \\
& \left.+20 d_{6}\right) u^{10}+2880\left(6860 d_{12}-3\left(20 d_{7}+35 d_{8}+42 d_{9}\right)\right) u^{11} \\
& -69984\left(5 d_{2}-7 d_{3}-33 d_{4}-21 d_{5}\right) u^{12}-14112\left(9 d_{10}-39 d_{11}\right. \\
& \left.+5 d_{6}\right) u^{13}+6720\left(1870 d_{12}-3\left(5 d_{7}+9 d_{8}+11 d_{9}\right)\right) u^{14} \\
& -4374\left(56 d_{2}-3\left(28 d_{3}+143 d_{4}+88 d_{5}\right)\right) u^{15}-1008\left(77 d_{10}\right. \\
& \left.-341 d_{11}+42 d_{6}\right) u^{16}+176\left(50050 d_{12}-378 d_{7}-693 d_{8}\right. \\
& \left.-858 d_{9}\right) u^{17}-2187\left(84 d_{2}-11\left(12 d_{3}+65 d_{4}+39 d_{5}\right)\right) u^{18}+O\left(u^{19}\right) \\
:= & \alpha_{0}+\alpha_{1} u+\cdots+\alpha_{18} u^{18}+O\left(u^{19}\right) .
\end{aligned}
$$

If $\bar{G}(u) \equiv 0$, then we have $\alpha_{0}=\alpha_{1}=\cdots=\alpha_{18}=0$, which turn out to be, by direct calculation, that $d_{0}=d_{1}=\cdots=d_{12}=0$. By the way, it is remarkable that we cannot get from $\alpha_{0}=\alpha_{1}=\cdots=\alpha_{12}=0$ that $d_{0}=\cdots=d_{12}=0$. This shows that our claim holds.

Since $g_{i}$ is an analytic function on $(0,1)$ for $i=0,1, \ldots, 12$, by applying the result of Lemma 4.5 of [5], we know that by suitable choice of $c_{0}, c_{1}, \ldots, c_{12}, c_{0} g_{0}(s)+c_{1} g_{1}(s)+\cdots+$ $c_{12} g_{12}(s)$ can have $0,1,2, \ldots, 12$ simple zeros in $(0,1)$.

Consequently, according to Theorem 4.3, there exist some coefficients $a_{i j}, b_{i j}(i+j=$ $0,1, \ldots, 7)$ such that system (5) has $0,1,2, \ldots, 12$ limit cycles. This completes the proof.

Remark 4.2. It seems very hard to find the smallest upper bound of the number of limit cycles of system (5) which emerge from the period annulus of the isochronous center for the case $n=2$. In fact, the expressions of the Wronskian determinants $W_{k}$ for $k \geq 9$ are too complicated to determine the number of simple zeros of them. On the other hand, by using the same method as the one in the proof of case $n=1$, we can find some coefficients $c_{0}, c_{1}, \ldots, c_{12}$ such that $c_{0} g_{0}(s)+c_{1} g_{1}(s)+\cdots+c_{12} g_{12}(s)$ has 12 simple zeros in a small interval $\left(s_{0}-\varepsilon, s_{0}+\varepsilon\right)$ and has extra zero in $\left(0, s_{0}-\varepsilon\right)$ with $s_{0}=7 / 10$. However, we cannot prove that the extra zero is simple.

\section{Appendix 1: The AVERAging THeORY of FirSt ORDeR}

In this appendix we present the basic results from the averaging theory that we shall need for proving the main results of this paper.

We consider the problem of the bifurcation of $T$-periodic solutions from differential systems of the form

$$
\mathbf{x}^{\prime}=F_{0}(t, \mathbf{x})+\varepsilon F_{1}(t, \mathbf{x})+\varepsilon^{2} F_{2}(t, \mathbf{x}, \varepsilon),
$$


with $\varepsilon=0$ to $\varepsilon \neq 0$ sufficiently small. Here the functions $F_{0}, F_{1}: \mathbb{R} \times \Omega \rightarrow \mathbb{R}^{n}$ and $F_{2}: \mathbb{R} \times \Omega \times\left(-\varepsilon_{0}, \varepsilon_{0}\right) \rightarrow \mathbb{R}^{n}$ are $\mathcal{C}^{2}$ functions, $T$-periodic in the first variable, and $\Omega$ is an open subset of $\mathbb{R}^{n}$. The main assumption is that the unperturbed system

$$
\mathbf{x}^{\prime}=F_{0}(t, \mathbf{x}),
$$

has a submanifold of dimension $n$ of periodic solutions. A solution of this problem is given using the averaging theory.

Let $\mathbf{x}(t, \mathbf{z}, \varepsilon)$ be the solution of the system (39) such that $\mathbf{x}(0, \mathbf{z}, \varepsilon)=\mathbf{z}$. We write the linearization of the unperturbed system along the periodic solution $\mathbf{x}(t, \mathbf{z}, 0)$ as

$$
\mathbf{y}^{\prime}=D_{\mathbf{x}} F_{0}(t, \mathbf{x}(t, \mathbf{z}, 0)) \mathbf{y}
$$

In what follows we denote by $M_{\mathbf{z}}(t)$ some fundamental matrix of the linear differential system (40).

We assume that there exists an open set $V$ with $\mathrm{Cl}(V) \subset \Omega$ such that for each $\mathbf{z} \in \mathrm{Cl}(V)$, $\mathbf{x}(t, \mathbf{z}, 0)$ is $T$-periodic. The set $\mathrm{Cl}(V)$ is isochronous for the system (38); i.e. it is a set formed only by periodic orbits, all of them having the same period. Then, an answer to the problem of the bifurcation of $T$-periodic solutions from the periodic solutions $\mathbf{x}(t, \mathbf{z}, 0)$ contained in $\mathrm{Cl}(V)$ is given in the following result.

Theorem 4.3 (Perturbations of an isochronous set). We assume that there exists an open and bounded set $V$ with $\mathrm{Cl}(V) \subset \Omega$ such that for each $\mathbf{z} \in \mathrm{Cl}(V)$, the solution $\mathbf{x}(t, \mathbf{z}, 0)$ is $T$-periodic, then we consider the function $\mathcal{F}: \mathrm{Cl}(V) \rightarrow \mathbb{R}^{n}$

$$
\mathcal{F}(\mathbf{z})=\int_{0}^{T} M_{\mathbf{z}}^{-1}(t) F_{1}(t, \mathbf{x}(t, \mathbf{z}, 0)) d t .
$$

If there exists $\alpha \in V$ with $\mathcal{F}(\alpha)=0$ and $\operatorname{det}((d \mathcal{F} / d \mathbf{z})(\alpha)) \neq 0$, then there exists a $T$ periodic solution $\mathbf{x}(t, \varepsilon)$ of system (38) such that $\mathbf{x}(0, \varepsilon) \rightarrow \alpha$ as $\varepsilon \rightarrow 0$.

Theorem 4.3 goes back to Malkin [14] and Roseau [17], for a shorter proof see [3].

\section{Appendix 2: Extended Complete Chebyshev system}

We say that the functions $\left(f_{0}, \ldots, f_{n}\right)$ defined on the interval $I$ form an Extended Chebyshev system or ET-system on $I$, if and only if, any nontrivial linear combination of these functions has at most $\mathrm{n}$ zeros counting their multiplicities and this number is reached. The functions $\left(f_{0}, \ldots, f_{n}\right)$ are an Extended Complete Chebyshev system or an ECT-system on $I$ if and only if for any $k \in\{0,1, \ldots, n\},\left(f_{0}, \ldots, f_{k}\right)$ form an ET-system.

Theorem 4.4. Let $f_{0}, \ldots, f_{n}$ be analytic functions defined on an open interval $I \subset \mathbb{R}$. Then $\left(f_{0}, \ldots, f_{n}\right)$ is an ECT-system on $I$ if and only if for each $k \in\{0,1, \ldots, n\}$ and all $y \in I$ the Wronskian

$$
W\left(f_{0}, \ldots, f_{k}\right)(y)=\left|\begin{array}{cccc}
f_{0}(y) & f_{1}(y) & \cdots & f_{k}(y) \\
f_{0}^{\prime}(y) & f_{1}^{\prime}(y) & \cdots & f_{k}^{\prime}(y) \\
\vdots & \vdots & \ddots & \vdots \\
f_{0}^{(k)}(y) & f_{1}^{(k)}(y) & \cdots & f_{k}^{(k)}(y)
\end{array}\right|
$$

is different from zero.

For a proof of Theorem 4.4 see [10]. 


\section{ACKNOWLEDGEMENTS}

This work was elaborated when H. Liang was visiting the Department of Mathematics of Universitat Autònoma de Barcelona. He is very grateful for the support and hospitality.

The first author is supported by the NSF of China (No. 11201086), the Foundation for Distinguished Young Talents in Higher Education of Guangdong, China (No. 2012LYM_0087) and the Excellent Young Teachers Training Program for colleges and universities of Guangdong Province, China (No. Yq2013107). The second and third authors are partially supported by the MINECO/FEDER grants MTM2008-03437, MTM201340998-P, and UNAB13-4E-1604; the AGAUR grant 2014 SGR568; an ICREA Academia grant; and the European grants FP7-PEOPLE-2012-IRSES 318999 and 316338.

\section{REFERENCES}

[1] M. Abramowitz, I. A. Stegun, Handbook of mathematical functions: with formulas, graphs, and mathematical tables, New York: Dover, 1965.

[2] V. I. Arnold, Ten problems, Adv. Soviet Math. 1 (1990), 1-8.

[3] A. Buica, J. P. Françoise, and J. Llibre, Periodic solutions of nonlinear periodic differential systems with a small parameter, Comm. Pure Appl. Anal. 6 (2006), 103-111.

[4] C. Christopher And C. Li, Limit cycles in differential equations, Birkhäuser, Boston, 2007.

[5] B. Coll, A. Gasull, and R. Prohens, Bifurcation of limit cycles from two families of centers, Dyn. Contin. Discrete Impuls. Syst. Ser. A Math. Anal., 12 (2005), 275-287.

[6] B. Coll, C. Li, And R. Prohens, Quadratic perturbations of a class of quadratic reversible systems with two centers. Disc. Contin. Dyn. Sys. 24 (2009), 699-729.

[7] D. Hilbert, Mathematische Probleme, Lecture, Second Internat. Congr. Math. (Paris, 1900), Nachr. Ges. Wiss. Göttingen Math. Phys. KL. (1900), 253-297; English transl., Bull. Amer. Math. Soc. 8 (1902), 437-479.

[8] Yu. Ilyashenko, Centennial history of Hilbert's 16th problem, Bull. (New Series) Amer. Math. Soc. 39 (2002), 301-354.

[9] V. Kaloshin, Around the Hilbert-Arnol'd problem. On finiteness in differential equations and Diophantine geometry, CRM Monograph Series 24, Edited by D. Schlomiuk, Amer. Math. Soc. Providence, RI, 2005, 111-162.

[10] J. Karlin And W. J. Studden, T-systems: with applications in analysis and statistics, Pure Appl. Math., Interscience Publishers, New York, London, Sidney, 1966.

[11] J. LI, Hilbert's 16th problem and bifurcations of planar polynomial vector fields, Internat. J. Bifur. Chaos Appl. Sci. Engrg. 13 (2003), 47-106.

[12] J. Llibre and L. A. Roberto, On the periodic solutions of a class of Duffing differential equations, Disc. Contin. Dyn. Sys. 33 (2013), 277-282.

[13] J. LlibRe and C. VAlls, Hopf bifurcation for some analytic differential systems in $R^{3}$ via averaging theory, Disc. Contin. Dyn. Sys. 30 (2011), 779-790.

[14] I. G. MaLkin, Some Problems of the theory of nonlinear oscillations, Gosudarstv. Izdat. Tehn-Teor. Lit. Moscow, 1956 (in Russian).

[15] D. D. Novaes and J. Torregrosa, On the extended Chebyshev systems with positive accuracy. Preprint. 2015.

[16] L. Peng And Y. LeI, The cyclicity of the period annulus of a quadratic reversible system with a hemicycle, Disc. Contin. Dyn. Sys. 30 (2011), 873-890.

[17] M. Roseau, Vibrations non linéaires et théorie de la stabilité, Springer Tracts in Natural Philosophy, Vol. 8, Springer, New York, 1985.

[18] J. Stoer and R. BuliRsch, Introduction to numerical analysis, Translated from the German by R. Bartels, W. Gautschi and C. Witzgall. Second edition. Texts in Applied Mathematics, 12. SpringerVerlag, New York, 1993.

[19] S. YAKOVEnKo, Qualitative theory of ordinary differential equations and tangential Hilbert 16th problem, CRM Monograph Series 24, Edited by D. Schlomiuk, Amer. Math. Soc. Providence, RI, 2005, 41-109. 
Department of Computer Science, Guangdong Polytechnic Normal University, Guangzhou, Guangdong 510665, P. R. China

E-mail address: haiihuaa@tom.com

Departament de Matemàtiques, Universitat Autònoma de Barcelona, 08193 Bellaterra, Barcelona, Catalonia, Spain

E-mail address: jllibre@mat.uab.cat

Departament de Matemàtiques, Universitat Autònoma de Barcelona, 08193 Bellaterra, Barcelona, Catalonia, Spain

E-mail address: torre@mat.uab.cat 